\title{
Feedback Stackelberg-Nash Equilibria in Mixed Leadership Games with an Application to Cooperative Advertising
}

\author{
Alain Bensoussan; Shaokuan Chen $†$ Anshuman Chutanił Suresh P. Sethi§̧ \\ Chi Chung Siu, Sheung Chi Phillip Yam॥
}

\begin{abstract}
In this paper we characterize the feedback equilibrium of a general infinite-horizon Stackelberg-Nash differential game where the roles of the players are mixed. By mixed we mean that one player is a leader on some decisions and a follower on other decisions. We prove a verification theorem that reduces the task of finding equilibrium strategies in functional spaces to two simple steps: First solving two static Nash games at the Hamiltonian level in a nested version and then solving the associated system of Hamilton-Jacobi-Bellman equations. As an application, we study a novel manufacturer-retailer cooperative advertising game where, in addition to the traditional setup into which the manufacturer subsidizes the retailer's advertising effort, we also allow the reverse support from the retailer to the manufacturer. In this representative case, we find an equilibrium that can be expressed by a solution of a set of algebraic equations. We then conduct an extensive numerical study to assess the impact of model parameters on the equilibrium.
\end{abstract}

Keywords: Stackelberg differential game, Nash differential game, feedback solution, cooperative advertising.

AMS Subject Classification: 49N90 Applications of optimal control and differential games; 49N70 Differential games; 90B60 Marketing, advertising.

\footnotetext{
*Naveen Jindal School of Management, The University of Texas at Dallas (Email: alain.bensoussan@utdallas.edu)

${ }^{\dagger}$ Target Corporation, USA (Email: Shaokuan.Chen@target.com)

${ }_{\ddagger}^{\ddagger}$ Nottingham University Business School (Email: anshuman.chutani@nottingham.ac.uk)

$\$$ Naveen Jindal School of Management, The University of Texas at Dallas (Email: sethi@utdallas.edu)

IDepartment of Mathematics and Statistics, School of Decision Sciences, The Hang Seng University of Hong Kong (Email: ccsiu@hsu.edu.hk)

"Department of Statistics, The Chinese University of Hong Kong (Email: scpyam@cuhk.edu.hk)
} 


\section{Introduction}

Heinrich Freiherr von Stackelberg [33] introduced a concept of equilibrium in games, which is now known as the Stackelberg equilibrium or the Stackelberg solution. In the Stackelberg two-person game, one player acts as the leader (she) while the other behaves as the follower (he). This sequential decision-making structure requires a solution method in which the leader incorporates the follower's best response into her action. Consequently, we can first solve the follower's optimization problem for any announced decision by the leader. This results in obtaining the follower's optimal response as a function of the leader's decision. Then the leader can solve her optimization problem that takes into account the follower's optimal response. This leads to the leader's optimal decision. Substituting the leader's optimal decision into the follower's response gives the follower's optimal decision. These two decisions taken together constitute a Stackelberg solution.

Later the concept of Stackelberg solution was extended to multi-period games. In dynamic settings, the dominance types of the leader over the follower as well as various information sets lead to a variety of equilibrium concepts. If in the beginning of the game the leader announces her whole strategy over the entire duration, then she dominates the follower globally and the corresponding solution is referred to as a global Stackelberg solution. If the leader acts ahead of the follower in every period (instant) based on the observed underlying state, the corresponding solution is referred to as a feedback solution. One can refer to [4] for the conceptualization of different equilibria in discrete-time and continuous-time deterministic settings. [17] and [24] survey various applications of dynamic Stackelberg game models in the operations management, supply chain management, and marketing literature.

In this paper we focus on obtaining feedback Stackelberg-Nash Equilibria in stochastic differential games. [18] initially formalized differential games and showed that the theory of optimal control can be adapted to study Nash differential games as dynamic extensions of the static Nash equilibria. Later, global Stackelberg equilibria were obtained by viewing the follower's Hamiltonian system as the leader's state equation for the deterministic case and the stochastic case (see, e.g., [4], [11], [25], [7]). For feedback Stackelberg equilibria, it was realized that one can also look for them in differential games as an appropriate limit of a sequence of dynamic discrete-time games, as the length of the period shrinks to zero [3]. However, the convergence of the solutions of the sequence of discrete-time games remains

a challenging open problem. Inspired by the results on feedback Nash equilibria where the feedbacks are defined at the Hamiltonian level and obtained through a system of HamiltonJacobi-Bellman (HJB) equations, we show that the feedback Stackelberg equilibria can also be obtained in a similar way: obtain the feedbacks of the static Stackelberg game at the 
Hamiltonian level and at each instant, and solve the resulting system of HJB equations.

This paper is devoted to a class of differential games where there are no clear leaders and followers as required in the original definition of a Stackelberg equilibrium. Indeed, in many realistic situations, a player may act as a leader for some decisions and as a follower for the remaining ones. For example, in the context of a manufacturer selling a product through a retailer, the manufacturer could act as a leader for advertising decisions, while the retailer could be the price leader. In this case, the manufacturer acts as a follower for making his wholesale price decision and the retailer acts as a follower for making his decision relating to local advertising of the product. Closer to home, it is possible to visualize a household scenario where the wife acts as leader for decisions relating to domestic activities, whereas the husband acts as the leader for budgetary or external decisions. Such games termed mixed leadership games were introduced in [5] and [6], along with a procedure to obtain open-loop equilibria. Open-loop solution is an example of a global solution, and for other types of global solutions, the readers may refer to [7]. While [5] and [6] deal only with open-loop solutions in mixed leadership games, this paper is devoted to feedback Stackelberg-Nash equilibria. It is important to emphasize that none of the global solutions are in general time-consistent, whereas a feedback solution is, which is an extremely desirable property. Without time consistency, one needs a commitment on the part of the leader to begin with, and perhaps a mechanism to enforce the commitment. Also, [5] derives necessary conditions in the form of a maximum principle, this paper, however, derives a sufficient condition in the form of a verification theorem.

In this paper, we develop a procedure to obtain a feedback equilibrium for mixed leadership games. Specifically, we formalize games where some decisions are announced by player 1 and some by player 2 simultaneously. Then, each player must obtain its optimal response for those decisions that are to be made by him in the role of the follower. Then, given these responses, the remaining decisions are made simultaneously. For convenience in exposition, we will partition all decisions into lead and follow-up decisions. The follow-up decisions are obtained simultaneously as the optimal responses to the lead decisions. Thus, this procedure involves both Nash and Stackelberg steps. And given the optimal responses, the lead decisions are made simultaneously as the Nash step. Thus, the game requires two Nash steps and one Stackelberg step in a nested fashion. In this paper we first provide the definition of a feedback equilibrium in this dynamic mixed leadership setting. We establish a verification theorem and show that finding such a dynamic equilibrium can actually be reduced to solving a mixed leadership static game at the Hamiltonian level and a system of HJB equations involving the above obtained static equilibrium. We then provide a detailed analysis of a representative example that admits a tractable, feedback Stackelberg-Nash equilibrium. It 
should be noted that standard fixed-point theories cannot be directly applied to our mixed leadership framework, and hence our analysis on this representative example marks as a first important step to tackle the fixed-point problems arising from the mixed leadership game problem.

More importantly, this representative example offers a new insight into the mixed leadership perspectives on the cooperative advertising decisions between a manufacturer and a retailer. In this respect, we investigate a cooperative advertising game in a manufacturerretailer supply chain where there are no clear leaders and followers in the following sense: Based on the observed market share, at each instant of time both players first simultaneously decide their support rates (lead decisions) of the other party's advertising effort, and then, based on the announced support rates as well as the market share determined according to an extension of the model in [27], simultaneously decide their own advertising effort (follow-up decisions). We apply the verification theorem and find an equilibrium at which the players' objective functions are affine functions of the market share. We derive a set of algebraic equations to characterize the equilibrium strategies. These algebraic equations are easy to solve numerically, and so we conduct a numerical analysis to examine the impact of model parameters on the players' decision variables, and compare our scenario with the traditional one-sided cooperative advertising game where only the manufacturer supports the retailer's advertising effort.

The Stackelberg setting has been found very useful in modeling many realistic situations in supply chains and marketing channels. Here we briefly review the related literature and distinguish our paper from existing works. [28] considered one lead firm and $N$ following firms which supply a homogeneous product noncooperatively to a market. The leader is called a Stackelberg firm and the followers are called Cournot firms as they compete with each other simultaneously in terms of quantities. The authors studied the existence and uniqueness of a Stackelberg-Nash-Cournot equilibrium and developed an algorithm to determine it. [34] studied a similar setting but assumed that the demand is unknown when the Stackelberg firm chooses the production level, whereas the Cournot firms make their decisions after the realization of the demand. [10] extended [34] to a multiple-leader Stackelberg-Nash-Cournot model and proposed a computational approach based on a sample average approximation to find the equilibrium. [1] considered competition in a supply chain where multiple manufacturers compete on supply quantities of a set of products and multiple risk-averse retailers compete on order quantities to satisfy customer demand. They examined the impact of asymmetry of manufacturers, retailers, and product assortment on supply chain efficiency. [12] analyzed sequential price competition in a multi-echelon supply chain where an arbitrary number of firms are allowed at each echelon. They characterized the equilibria, provided 
a computational scheme to find them, and showed comparative statics results with respect to model parameters. However, the models reviewed above involving both horizonal and vertical competitions are static games in operations management. Our paper complements the existing literature by addressing Stackelberg-Nash games in a dynamic setting.

There are some studies in marketing literature which consider dynamic game theoretic models in cooperative advertising; see for e.g., [19, 20, 21], [23], [16], [29], [14, 15], [8, 9]. The readers are referred to [17], [2], [22], and [24], for extensive surveys on cooperative advertising models. However, there are key differences between these papers and the application in this paper. These papers consider advertising support in only one direction (manufacturer to retailer), i.e., only one party supports the other in its advertising efforts. Whereas in our model, both the manufacturer and the retailer make advertising efforts, and both may support each other by sharing a fraction of the other's advertising cost. Furthermore, these papers model the problem in a classical pure Stackelberg or pure Nash framework, whereas we consider a mixed leadership game in a feedback Stackelberg-Nash framework, which has not been studied earlier to the best of our knowledge.

The paper is organized as follows. We first formulate a general mixed leadership differential game and give the feedback equilibrium definition in Section 2. We characterize the feedback Stackelberg-Nash equilibrium in terms of the solutions to a system of coupled non-linear ordinary differential equations and provide a corresponding verification theorem in Section 2.2. Based on this result, we study a cooperative advertising game in a manufacturerretailer supply chain in Section 3, and obtain a system of algebraic equations for a specific equilibrium where the players' profits are affine functions of the market share. In Section 4 , we numerically examine the impact of model parameters on the equilibrium behavior and the resulting managerial insights. We conclude our paper in Section 5.

\section{A Mixed-Leadership Differential Game Model}

\subsection{Model and problem formulation}

Consider a game model with two players 1 and 2. The game model under consideration is given by a state equation

$$
\mathrm{d} x(t)=f\left(x(t), u_{1}(t), v_{1}(t), u_{2}(t), v_{2}(t)\right) \mathrm{d} t+\sigma(x(t)) \mathrm{d} W(t), \quad x(0)=x,
$$

where $x$ is an initial state known by both players, $f: \mathbb{R} \times \mathbb{U}_{1} \times \mathbb{V}_{1} \times \mathbb{U}_{2} \times \mathbb{V}_{2} \rightarrow \mathbb{R}$ and $\sigma: \mathbb{R} \rightarrow \mathbb{R}$ are the drift and the volatility functions of $x$, respectively; $W$ is an one-dimensional standard 
Brownian motion defined on a complete probability space $(\Omega, \mathcal{F}, \mathbb{P})$; and $\left(u_{i}(t), v_{i}(t)\right) \in$ $\mathbb{U}_{i} \times \mathbb{V}_{i} \subseteq \mathbb{R} \times \mathbb{R}, t \in[0,+\infty), i=1,2$, are the decision variables of the two players.

To ensure that the state equation $x(t)$ in (1) yields a unique state trajectory, we assume that $f$ and $\sigma$ satisfy the conditions below (see, e.g. [13]):

(i) For all $\left(u_{i}, v_{i}\right) \in \mathbb{U}_{i} \times \mathbb{V}_{i}$ with $i=1,2, f$ is locally Lipschitz continuous in $x$ and $\sigma$ is locally Lipschitz continuous in $x$.

(ii) $f$ is continuously differentiable in $\left(u_{i}, v_{i}\right) \in \mathbb{U}_{i} \times \mathbb{V}_{i}$ with $i=1,2$.

For $i=1,2$, denote $J_{i}^{x}$ to be an expected profit function of player 1

$$
J_{i}^{x}\left(u_{1}, v_{1}, u_{2}, v_{2}\right)=\mathbb{E}\left[\int_{0}^{\infty} \mathrm{e}^{-\rho t} l_{i}\left(x(t), u_{1}(t), v_{1}(t), u_{2}(t), v_{2}(t)\right) \mathrm{d} t \mid x(0)=x\right],
$$

where $l_{i}$ is a continuously differentiable function in $\left(u_{i}, v_{i}\right) \in \mathbb{U}_{i} \times \mathbb{V}_{i}$ with $i=1,2$ that represents the profit function of player $i$.

In view of the objective functions of the two players in (2), we define the admissible strategy space $\mathcal{U}_{i} \times \mathcal{V}_{i} \subseteq \mathbb{U}_{i} \times \mathbb{V}_{i}$, for $i=1,2$, to be the set of strategies such that

$\mathcal{U}_{i} \triangleq\left\{u_{i} \mid u_{i}: \mathbb{R} \rightarrow \mathbb{U}_{i}\right.$ and $u_{i}(x)$ is locally Lipschitz continuous in $\left.x\right\}$, $\mathcal{V}_{i} \triangleq\left\{v_{i} \mid v_{i}: \mathbb{R} \times \mathbb{U}_{1} \times \mathbb{U}_{2} \rightarrow \mathbb{V}_{i}\right.$ and $v_{i}\left(x, \mu_{1}, \mu_{2}\right)$ is locally Lipschitz continuous in $\left.\left(x, \mu_{1}, \mu_{2}\right)\right\}$, and the following integrability condition is satisfied: for all $\left(u_{i}, v_{i}\right) \in \mathcal{U}_{i} \times \mathcal{V}_{i}, i=1,2$,

$$
\mathbb{E}\left[\int_{0}^{\infty} \mathrm{e}^{-\rho s}\left|l_{i}\left(x\left(s ; u_{1}, v_{1}, u_{2}, v_{2}\right), u_{1}, v_{1}, u_{1}, u_{2}, v_{2}\right)\right| \mathrm{d} s\right]<\infty
$$

where $u_{i}, v_{i}$ are evaluated at $x\left(s ; u_{1}, v_{1}, u_{2}, v_{2}\right)$ with the forms

$$
\begin{gathered}
u_{i}\left(x\left(s ; u_{1}, v_{1}, u_{2}, v_{2}\right)\right) \\
v_{i}\left(x\left(s ; u_{1}, v_{1}, u_{2}, v_{2}\right), u_{1}\left(x\left(s ; u_{1}, v_{1}, u_{2}, v_{2}\right)\right), u_{2}\left(x\left(s ; u_{1}, v_{1}, u_{2}, v_{2}\right)\right)\right) .
\end{gathered}
$$

This admissible strategy space $\mathcal{U}_{i} \times \mathcal{V}_{i} \subseteq \mathbb{U}_{i} \times \mathbb{V}_{i}$, for $i=1$, 2, indicates that the underlying information structure is of a feedback form for player $i$, in which $u_{i}$ is the lead decision variable and $v_{i}$ is the follow decision variable.

We are now in position to define an optimization problem of each player $i$, for $i=1,2$ :

Problem 2.1. Given the strategy pair of the other player $j,\left(u_{j}, v_{j}\right) \in \mathcal{U}_{j} \times \mathcal{V}_{j}$, for $j \neq i \in$ $\{1,2\}$, player $i$ chooses her strategy pair $\left(u_{i}, v_{i}\right) \in \mathcal{U}_{i} \times \mathcal{V}_{i}$ to maximize her expected profit 
function $J_{i}^{x}$ in (2) subject to

$$
\left\{\begin{aligned}
\mathrm{d} x(s)= & f\left(x(s), u_{1}(x(s)), v_{1}\left(x(s), u_{1}(x(s)), u_{2}(x(s))\right), u_{2}(x(s)), v_{2}\left(x(s), u_{1}(x(s)), u_{2}(x(s))\right)\right) \mathrm{d} s \\
& +\sigma(x(s)) \mathrm{d} W(s), \\
x(0)= & x .
\end{aligned}\right.
$$

When there is no ambiguity about the chosen strategies $\left(u_{1}, v_{1}, u_{2}, v_{2}\right)$ from the context, we will hereafter use an abbreviated notation $x(\cdot)$ for $x\left(\cdot ; u_{1}, v_{1}, u_{2}, v_{2}\right)$ and omit the arguments of the strategies $u_{i}$ and $v_{i}$ in $f$ and $l_{i}, i=1,2$, for the sake of notational convenience. In view of Problem 2.1, we now provide a rigorous definition of the equilibrium analyzed in our paper.

Definition 2.1. Two pairs of strategies $\left(u_{i}^{*}, v_{i}^{*}\right) \in \mathcal{U}_{i} \times \mathcal{V}_{i}, i=1,2$, are called a feedback Stackelberg-Nash equilibrium if the following holds:

$$
\begin{aligned}
& J_{1}^{x}\left(u_{1}^{*}(\cdot), v_{1}^{*}\left(\cdot, u_{1}^{*}(\cdot), u_{2}^{*}(\cdot)\right), u_{2}^{*}(\cdot), v_{2}^{*}\left(\cdot, u_{1}^{*}(\cdot), u_{2}^{*}(\cdot)\right)\right) \\
\geq & J_{1}^{x}\left(u_{1}(\cdot), v_{1}\left(\cdot, u_{1}(\cdot), u_{2}^{*}(\cdot)\right), u_{2}^{*}(\cdot), v_{2}^{*}\left(\cdot, u_{1}(\cdot), u_{2}^{*}(\cdot)\right)\right), \forall\left(u_{1}, v_{1}\right) \in \mathcal{U}_{1} \times \mathcal{V}_{1}, \forall x \in \mathbb{R} \\
& J_{2}^{x}\left(u_{1}^{*}(\cdot), v_{1}^{*}\left(\cdot, u_{1}^{*}(\cdot), u_{2}^{*}(\cdot)\right), u_{2}^{*}(\cdot), v_{2}^{*}\left(\cdot, u_{1}^{*}(\cdot), u_{2}^{*}(\cdot)\right)\right) \\
\geq & J_{2}^{x}\left(u_{1}^{*}(\cdot), v_{1}^{*}\left(\cdot, u_{1}^{*}(\cdot), u_{2}(\cdot)\right), u_{2}(\cdot), v_{2}\left(\cdot, u_{1}^{*}(\cdot), u_{2}(\cdot)\right)\right), \forall\left(u_{2}, v_{2}\right) \in \mathcal{U}_{2} \times \mathcal{V}_{2}, \forall x \in \mathbb{R} .
\end{aligned}
$$

In the above definition, we can see that in equilibrium no player can benefit from unilaterally changing its own strategy. In our mixed leadership feedback game, both players are leaders in $u$ decisions, and followers in $v$ decisions. In other words, at each instant of time, first player 1 (P1) decides $u_{1}$ and player 2 (P2) decides $u_{2}$, simultaneously. Then P1 and P2 follow with their decisions $v_{1}$ and $v_{2}$ at each instant of time, respectively and simultaneously. At the level of $u$ decisions, P1 and P2 play a feedback Nash game. At the level of $v$ decisions, P1 and P2 also play a feedback Nash game, with the additional information of the instantaneous actions from the level of $u$ decisions. From a vertical view of the two Nash games, they are played with hierarchy and therefore constitute a feedback Stackelberg game. In addition, since each player is both a leader in $u$ decision and a follower in $v$ decision, the roles of players in the game are mixed. For the above two reasons, we term this game a mixed feedback Stackelberg-Nash game, or mixed leadership game. To formalize the study of the mixed leadership game, we first focus on equilibrium characterization. Since both feedback Nash game and feedback Stackelberg game can be solved at the level of Hamiltonian, we first introduce the related Hamiltonians for the mixed Stackelberg-Nash game in Section 2.2. 


\subsection{Characterization of a Feedback Stackelberg-Nash Equilibrium}

In this section we characterize the mixed feedback Stackelberg-Nash equilibrium in terms of the solution to a system of ordinary differential equations (ODEs) involving two static Nash games and a Stackelberg game at the level of Hamiltonian. To this end, we first introduce the Hamiltonians for both players. For $p_{i} \in \mathbb{R}$, we define the Hamiltonian functions as follows

$$
\begin{aligned}
& H_{1}\left(x, \mu_{1}, \nu_{1}, \mu_{2}, \nu_{2}, p_{1}\right) \triangleq l_{1}\left(x, \mu_{1}, \nu_{1}, \mu_{2}, \nu_{2}\right)+p_{1} \cdot f\left(x, \mu_{1}, \nu_{1}, \mu_{2}, \nu_{2}\right), \\
& H_{2}\left(x, \mu_{1}, \nu_{1}, \mu_{2}, \nu_{2}, p_{2}\right) \triangleq l_{2}\left(x, \mu_{1}, \nu_{1}, \mu_{2}, \nu_{2}\right)+p_{2} \cdot f\left(x, \mu_{1}, \nu_{1}, \mu_{2}, \nu_{2}\right)
\end{aligned}
$$

For a given pair of actions $\left(\mu_{1}, \mu_{2}\right) \in \mathcal{U}_{1} \times \mathcal{U}_{2}$, we first consider a static Nash game with $\nu_{1}$ and $\nu_{2}$ as the decision variables at the level of Hamiltonian. We suppose that we can get a unique solution $\left(\Gamma_{1}^{\nu}\left(x, \mu_{1}, \mu_{2}, p_{1}, p_{2}\right), \Gamma_{2}^{\nu}\left(x, \mu_{1}, \mu_{2}, p_{1}, p_{2}\right)\right)$ from the equations

$$
\left\{\begin{array}{l}
\nu_{1}=\arg \max _{\nu_{1} \in \mathcal{V}_{1}} H_{1}\left(x, \mu_{1}, \nu_{1}, \mu_{2}, \nu_{2}, p_{1}\right) \\
\nu_{2}=\arg \max _{\nu_{2} \in \mathcal{V}_{2}} H_{2}\left(x, \mu_{1}, \nu_{1}, \mu_{2}, \nu_{2}, p_{2}\right)
\end{array}\right.
$$

Taking into account the response at the $v$ level, we substitute $\left(\Gamma_{1}^{\nu}, \Gamma_{2}^{\nu}\right)$ into $H_{1}$ and $H_{2}$, and solve the following static Nash game with $\mu_{1}$ and $\mu_{2}$ as decision variables at the level of Hamiltonian:

$$
\left\{\begin{array}{l}
\mu_{1}=\arg \max _{\mu_{1} \in \mathcal{U}_{1}} H_{1}\left(x, \mu_{1}, \Gamma_{1}^{\nu}\left(x, \mu_{1}, \mu_{2}, p_{1}, p_{2}\right), \mu_{2}, \Gamma_{2}^{\nu}\left(x, \mu_{1}, \mu_{2}, p_{1}, p_{2}\right), p_{1}\right), \\
\mu_{2}=\arg \max _{\mu_{2} \in \mathcal{U}_{2}} H_{2}\left(x, \mu_{1}, \Gamma_{1}^{\nu}\left(x, \mu_{1}, \mu_{2}, p_{1}, p_{2}\right), \mu_{2}, \Gamma_{2}^{\nu}\left(x, \mu_{1}, \mu_{2}, p_{1}, p_{2}\right), p_{2}\right) .
\end{array}\right.
$$

We assume that we have a unique solution $\left(\Gamma_{1}^{\mu}\left(x, p_{1}, p_{2}\right), \Gamma_{2}^{\mu}\left(x, p_{1}, p_{2}\right)\right)$ of the equations (7).

Remark 2.1. In general, it is typically difficult to establish a set of sufficient conditions to guarantee the existence of solutions to the fixed points problems in (6) and (7). The main difficulty stems from the fact that (6) and (7) are nested and must be solved hierarchically. Indeed, one begins by solving the fixed-point equations (6) in terms of $\mu_{1} \in \mathcal{U}_{1}, \mu_{2} \in \mathcal{U}_{2}$ and $p_{1}, p_{2}>0$ and obtain $\left(\Gamma_{1}^{\nu}, \Gamma_{2}^{\nu}\right)$. By assuming certain regularity properties of $\left(\Gamma_{1}^{\nu}, \Gamma_{2}^{\nu}\right)$, one then proceeds to solve the fixed-point equations in (7) in terms of $p_{1}, p_{2}>0$ to obtain $\left(\Gamma_{1}^{\mu}\left(x, p_{1}, p_{2}\right), \Gamma_{2}^{\mu}\left(x, p_{1}, p_{2}\right)\right)$.

Fortunately, there exists a representative example of the mixed leadership games in which the fixed point problems in (6) and (7) admit tractable solutions. We shall provide a detailed analysis of this representative example in Section 3.

With these notations, we now provide a verification theorem to show that the solutions 
in (6) and (7) constitute a feedback Stackelberg-Nash equilibrium. We should stress that we do not address the issue of the uniqueness of such an equilibrium. In general, multiple equilibria could occur in differential games including mixed leadership games. Denote

$$
\left\{\begin{array}{l}
V_{1}(x) \triangleq J_{1}^{x}\left(u_{1}^{*}(\cdot), v_{1}^{*}\left(\cdot, u_{1}^{*}(\cdot), u_{2}^{*}(\cdot)\right), u_{2}^{*}(\cdot), v_{2}^{*}\left(\cdot, u_{1}^{*}(\cdot), u_{2}^{*}(\cdot)\right)\right) \\
V_{2}(x) \triangleq J_{2}^{x}\left(u_{1}^{*}(\cdot), v_{1}^{*}\left(\cdot, u_{1}^{*}(\cdot), u_{2}^{*}(\cdot)\right), u_{2}^{*}(\cdot), v_{2}^{*}\left(\cdot, u_{1}^{*}(\cdot), u_{2}^{*}(\cdot)\right)\right)
\end{array}\right.
$$

where $\left(u_{i}^{*}, v_{i}^{*}\right) \in \mathcal{U}_{i} \times \mathcal{V}_{i}$, for $i=1,2$, is the feedback Nash-Stackelberg equilibrium in Definition 2.1. We have the following verification theorem.

Theorem 2.1 (Verification Theorem). Let $w_{i}$, for $i=1,2$, be a function in $\mathcal{C}^{2}(\mathbb{R})$ that satisfies a polynomial growth condition.

(i) Suppose that

$$
\left\{\begin{aligned}
0 \leq \rho w_{1}(x)-\frac{1}{2} \sigma^{2}(x) w_{1}^{\prime \prime}(x)-\sup _{\left(u_{1}, v_{1}\right) \in \mathcal{U}_{1} \times \mathcal{V}_{1}} & H_{1}\left(x, u_{1}(x), v_{1}\left(x, u_{1}(x), u_{2}^{*}(x)\right),\right. \\
& \left.u_{2}^{*}(x), v_{2}^{*}\left(x, u_{1}(x), u_{2}^{*}(x)\right), w_{1}^{\prime}(x)\right), \\
0 \leq \rho w_{2}(x)-\frac{1}{2} \sigma^{2}(x) w_{2}^{\prime \prime}(x)-\sup _{\left(u_{2}, v_{2}\right) \in \mathcal{U}_{2} \times \mathcal{V}_{2}} & H_{2}\left(x, u_{1}^{*}(x), v_{1}^{*}\left(x, u_{1}^{*}(x), u_{2}(x)\right),\right. \\
& \left.u_{2}(x), v_{2}\left(x, u_{1}^{*}(x), u_{2}(x)\right), w_{2}^{\prime}(x)\right),
\end{aligned}\right.
$$

where

$$
\begin{array}{r}
\left\{\begin{array}{l}
u_{1}^{*}(x) \triangleq \Gamma_{1}^{\mu}\left(x, w_{1}^{\prime}, w_{2}^{\prime}\right), \\
u_{2}^{*}(x) \triangleq \Gamma_{2}^{\mu}\left(x, w_{1}^{\prime}, w_{2}^{\prime}\right)
\end{array}\right. \\
\left\{\begin{array}{l}
v_{1}^{*}\left(x, u_{1}^{*}(x), u_{2}(x)\right) \triangleq \Gamma_{1}^{\nu}\left(x, u_{1}^{*}(x), u_{2}(x), w_{1}^{\prime}, w_{2}^{\prime}\right), \\
v_{2}^{*}\left(x, u_{1}(x), u_{2}^{*}(x)\right) \triangleq \Gamma_{2}^{\nu}\left(x, u_{1}(x), u_{2}^{*}(x), w_{1}^{\prime}, w_{2}^{\prime}\right),
\end{array}\right.
\end{array}
$$

with

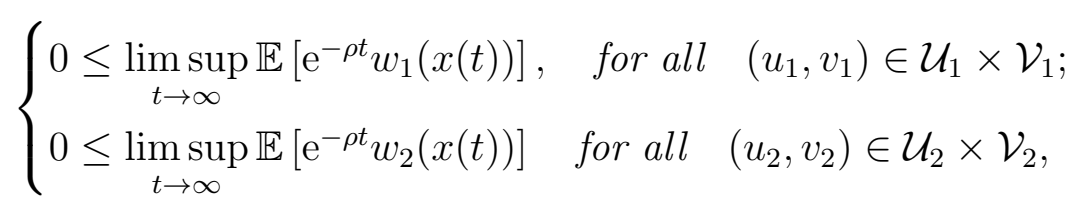

Then, $w_{1} \geq V_{1}$ and $w_{2} \geq V_{2}$.

(ii) Suppose further that, for all $x \in \mathbb{R}$, there exist $\left(u_{1}^{*}, v_{1}^{*}\right) \in \mathcal{U}_{1} \times \mathcal{V}_{1}$ and $\left(u_{2}^{*}, v_{2}^{*}\right) \in \mathcal{U}_{2} \times \mathcal{V}_{2}$, 
where

$$
\begin{gathered}
\left\{\begin{array}{l}
u_{1}^{*}(x) \triangleq \Gamma_{2}^{\mu}\left(x, w_{1}^{\prime}, w_{2}^{\prime}\right), \\
u_{2}^{*}(x) \triangleq \Gamma_{2}^{\mu}\left(x, w_{1}^{\prime}, w_{2}^{\prime}\right) ;
\end{array}\right. \\
\left\{\begin{array}{l}
v_{1}^{*}\left(x, u_{1}^{*}(x), u_{2}^{*}(x)\right) \triangleq \Gamma_{1}^{\nu}\left(x, u_{1}^{*}(x), u_{2}^{*}(x), w_{1}^{\prime}, w_{2}^{\prime}\right), \\
v_{2}^{*}\left(x, u_{1}^{*}(x), u_{2}^{*}(x)\right) \triangleq \Gamma_{2}^{\nu}\left(x, u_{1}^{*}(x), u_{2}^{*}(x), w_{1}^{\prime}, w_{2}^{\prime}\right),
\end{array}\right.
\end{gathered}
$$

such that

$$
\left\{\begin{array}{r}
0=\rho w_{1}(x)-\frac{1}{2} \sigma^{2}(x) w_{1}^{\prime \prime}(x)-H_{1}\left(x, u_{1}^{*}(x), v_{1}^{*}\left(x, u_{1}^{*}(x), u_{2}^{*}(x)\right),\right. \\
\left.u_{2}^{*}(x), v_{2}^{*}\left(x, u_{1}^{*}(x), u_{2}^{*}(x)\right), w_{1}^{\prime}(x)\right), \\
0=\rho w_{2}(x)-\frac{1}{2} \sigma^{2}(x) w_{1}^{\prime \prime}(x)-H_{2}\left(x, u_{1}^{*}(x), v_{1}\left(x, u_{1}^{*}(x), u_{2}^{*}(x)\right),\right. \\
\left.u_{2}^{*}(x), v_{2}^{*}\left(x, u_{1}^{*}(x), u_{2}^{*}(x)\right), w_{2}^{\prime}(x)\right),
\end{array}\right.
$$

satisfying

$$
\left\{\begin{array}{l}
\liminf _{t \rightarrow \infty} \mathbb{E}\left[\mathrm{e}^{-\rho t} w_{1}\left(x^{*}(t)\right)\right] \leq 0 \\
\liminf \operatorname{in}_{t \rightarrow \infty} \mathbb{E}\left[\mathrm{e}^{-\rho t} w_{2}\left(x^{*}(t)\right)\right] \leq 0
\end{array}\right.
$$

Then $w_{1}(x)=V_{1}(x), w_{2}=V_{2}(x)$, and that $\left\{\left(u_{1}^{*}, v_{1}^{*}\right),\left(u_{2}^{*}, v_{2}^{*}\right)\right\}$ is a feedback StackelbergNash equilibrium to Problem 2.1.

Proof. By symmetry, it suffices to focus on the case of $w_{1}$. We shall suppress the arguments in $u_{i}, v_{i}, i=1 ; 2$, for notational convenience.

(i) Suppose that player 1 adopts an arbitrary pair of strategies $\left(u_{1}, v_{1}\right) \in \mathcal{U}_{1} \times \mathcal{V}_{1}$ and player 2 adopts the pair of strategies $\left(u_{2}^{*}, v_{2}^{*}\right)$ defined in (10) and (11). Let $B_{r}(x)$ represent an open ball of radius $r$, centered at $x$, i.e.,

$$
B_{r}(x) \triangleq\{y \in \mathbb{R}|| x-y \mid<r\}
$$

and let $\tau_{r}$ be the first exit time of $x(\cdot)$ from $B_{r}(x)$, i.e.,

$$
\tau_{r} \triangleq \inf \left\{s \mid x(s) \notin B_{r}(x), s \geq 0\right\}
$$


with the convention that $\inf \emptyset=\infty$ if $x(\cdot)$ does not exit from $B_{r}(x)$.

Applying Itô's formula to $\mathrm{e}^{-\rho s} w_{1}(x(s))$, integrating from 0 to $\tau_{r} \wedge t$, and taking expectation, we get

$$
\begin{aligned}
w_{1}(x)= & \mathbb{E}\left[\mathrm{e}^{-\rho\left(\tau_{r} \wedge t\right)} w_{1}\left(x\left(\tau_{r} \wedge t\right)\right)\right]-\mathbb{E}\left[\int _ { 0 } ^ { \tau _ { r } \wedge t } \mathrm { e } ^ { - \rho s } \left(-\rho w_{1}(x(s))+\frac{1}{2} \sigma^{2}(x(s)) w_{1}^{\prime \prime}(x(s))\right.\right. \\
& \left.\left.+f\left(x(s), u_{1}, v_{1}, u_{2}^{*}, v_{2}^{*}\right) w_{1}^{\prime}(x(s))\right) \mathrm{d} s\right] \\
= & \mathbb{E}\left[\mathrm{e}^{-\rho\left(\tau_{r} \wedge t\right)} w_{1}\left(x\left(\tau_{r} \wedge t\right)\right)\right]-\mathbb{E}\left[\int _ { 0 } ^ { \tau _ { r } \wedge t } \mathrm { e } ^ { - \rho s } \left(-\rho w_{1}(x(s))+\frac{1}{2} \sigma^{2}(x(s)) w_{1}^{\prime \prime}(x(s))\right.\right. \\
& \left.\left.+f\left(x(s), u_{1}, v_{1}, u_{2}^{*}, v_{2}^{*}\right) w_{1}^{\prime}(x(s))+l_{1}\left(x(s), u_{1}, v_{1}, u_{2}^{*}, v_{2}^{*}\right)\right) \mathrm{d} s\right] \\
& +\mathbb{E}\left[\int_{0}^{\tau_{r} \wedge t} \mathrm{e}^{-\rho s} l_{1}\left(x(s), u_{1}, v_{1}, u_{2}^{*}, v_{2}^{*}\right) \mathrm{d} s\right] \\
= & \mathbb{E}\left[\mathrm{e}^{-\rho\left(\tau_{r} \wedge t\right)} w_{1}\left(x\left(\tau_{r} \wedge t\right)\right)\right]-\mathbb{E}\left[\int _ { 0 } ^ { \tau _ { r } \wedge t } \mathrm { e } ^ { - \rho s } \left(-\rho w_{1}(x(s))+\frac{1}{2} \sigma^{2}(x(s)) w_{1}^{\prime \prime}(x(s))\right.\right. \\
& \left.+H_{1}\left(x(s), u_{1}, v_{1}, u_{2}^{*}, v_{2}^{*}, w_{1}^{\prime}(x(s))\right) \mathrm{d} s\right]+\mathbb{E}\left[\int_{0}^{\tau_{r} \wedge t} \mathrm{e}^{-\rho s} l_{1}\left(x(s), u_{1}, v_{1}, u_{2}^{*}, v_{2}^{*}\right) \mathrm{d} s\right] \\
\geq & \mathbb{E}\left[\mathrm{e}^{-\rho\left(\tau_{r} \wedge t\right)} w_{1}\left(x\left(\tau_{r} \wedge t\right)\right)\right]+\mathbb{E}\left[\int_{0}^{\tau_{r} \wedge t} \mathrm{e}^{-\rho s} l_{1}\left(x(s), u_{1}, v_{1}, u_{2}^{*}, v_{2}^{*}\right) \mathrm{d} s\right],
\end{aligned}
$$

where the last inequality follows from (9).

By the polynomial growth condition on $w_{i}$, for $i=1,2$, and the integrability condition in (3), we can apply the dominated convergence theorem and send $r$ to infinity to obtain

$$
w_{1}(x) \geq \mathbb{E}\left[\mathrm{e}^{-\rho t} w_{1}(x(t))\right]+\mathbb{E}\left[\int_{0}^{t} \mathrm{e}^{-\rho s} l_{1}\left(x(s), u_{1}, v_{1}, u_{2}^{*}, v_{2}^{*}\right) \mathrm{d} s\right]
$$

for all $\left(u_{1}, v_{1}\right) \in \mathcal{U}_{1} \times \mathcal{V}_{1}$. Using (12), we can apply the dominated convergence theorem again and sending $t$ to infinity to obtain

$$
w_{1}(x) \geq \mathbb{E}\left[\int_{0}^{\infty} \mathrm{e}^{-\rho s} l_{1}\left(x(s), u_{1}, v_{1}, u_{2}^{*}, v_{2}^{*}\right) \mathrm{d} s\right]=J_{1}^{x}\left(x, u_{1}, v_{1}, u_{2}^{*}, v_{2}^{*}\right)
$$


for all $\left(u_{1}, v_{1}\right) \in \mathcal{U}_{1} \times \mathcal{V}_{1}$. Therefore, it follows that

$$
w_{1}(x) \geq \sup _{u_{1} \in \mathcal{U}_{1}, v_{1} \in \mathcal{V}_{1}} J_{1}^{x}\left(x, u_{1}, v_{1}, u_{2}^{*}, v_{2}^{*}\right)=V_{1}(x)
$$

(ii) Repeat the exact arguments as in (i) and observe that the equilibrium condition in (15) is obtained at $\left(u_{1}^{*}, v_{1}^{*}\right)$ in (13)-(14). Consequently, we have

$$
\mathbb{E}\left[\mathrm{e}^{-\rho t} w_{1}\left(x^{*}(t)\right)\right]=w_{1}(x)-\mathbb{E}\left[\int_{0}^{t} \mathrm{e}^{-\rho s} l_{1}\left(x^{*}(s), u_{1}^{*}, v_{1}^{*}, u_{2}^{*}, v_{2}^{*}\right) \mathrm{d} s\right]
$$

Due to (16), we can apply the dominating convergence theorem again and send $t$ to infinity to obtain

$$
w_{1}(x) \leq \mathbb{E}\left[\int_{0}^{\infty} \mathrm{e}^{-\rho s} l_{1}\left(x^{*}(s), u_{1}^{*}, v_{1}^{*}, u_{2}^{*}, v_{2}^{*}\right) \mathrm{d} s\right]=J_{1}^{x}\left(x, u_{1}^{*}, v_{1}^{*}, u_{2}^{*}, v_{2}^{*}\right)=V_{1}(x)
$$

Finally, combining the right-hand sides of (18) and (21), it follows readily that

$$
J_{1}^{x}\left(x, u_{1}^{*}, v_{1}^{*}, u_{2}^{*}, v_{2}^{*}\right) \geq J_{1}^{x}\left(x, u_{1}, v_{1}, u_{2}^{*}, v_{2}^{*}\right)
$$

from which it follows readily that $\left(u_{1}^{*}, v_{1}^{*}\right)$ in (13)-(14) constitute a Nash-Stackelberg equilibrium.

A direct consequence of Theorem 2.1 is that the value functions $V_{1}, V_{2} \in \mathcal{C}^{2}(\mathbb{R})$ of players 1 and 2 at the mixed Nash-Stackelberg equilibrium $\left(u_{i}^{*}, v_{i}^{*}\right)$, for $i=1,2$, if it exists, are the solutions to the following system of coupled ODEs:

$$
\left\{\begin{aligned}
0=\rho V_{1}(x)-\frac{1}{2} \sigma^{2}(x) V_{1}^{\prime \prime}(x)-H_{1}\left(x, u_{1}^{*}(x), v_{1}^{*}\left(x, u_{1}^{*}(x), u_{2}^{*}(x)\right)\right. & \\
& \left.u_{2}^{*}(x), v_{2}^{*}\left(x, u_{1}^{*}(x), u_{2}^{*}(x)\right), V_{1}^{\prime}(x)\right) \\
0=\rho V_{2}(x)-\frac{1}{2} \sigma^{2}(x) V_{2}^{\prime \prime}(x)-H_{2}\left(x, u_{1}^{*}(x),\right. & v_{1}^{*}\left(x, u_{1}^{*}(x), u_{2}^{*}(x)\right) \\
& \left.u_{2}^{*}(x), v_{2}^{*}\left(x, u_{1}^{*}(x), u_{2}^{*}(x)\right), V_{2}^{\prime}(x)\right) .
\end{aligned}\right.
$$

Due to the feedback feature of the mixed Nash-Stackelberg equilibrium $\left(u_{i}^{*}, v_{i}^{*}\right)$, for $i=1,2$, in (6)-(7), the system of coupled ODEs in (23) is highly non-linear in general and its solution is typically difficult to obtain. Nonetheless, we now turn to a representative example in which 
solving the system of coupled ODEs in (23) can be reduced to solving a system of algebraic equations.

\section{A Model of Cooperative Advertising}

In this section, we consider a representative example of the mixed leadership game that admits tractable solutions in (6) and (7). More importantly, this representative example has an important application in cooperative adverising decisions between a manufacturer and a retailer. Specifically, we consider a manufacturer-retailer supply chain in a mature product category where sales, expressed as a fraction of the potential market, are positively influenced through advertising spending by the supply chain partners. We use the mixed leadership game theoretic framework developed in Sections 2-3 to study advertising cooperation between the manufacturer and the retailer. The advertising is done at the national level (e.g., TV ads broadcasted nationally during prime time, in national print media, etc.), and at the local level (e.g., advertisements in local print media, local radio and TV stations, pamphlets, promotions, etc.) We assume that the manufacturer decides the national advertising effort whereas the local advertising effort is decided by the retailer. Both types of advertising have stimulating effects on the sales, to be expressed shortly in the state dynamics of our model. The manufacturer, or the retailer, or both may decide to support the other in its advertising effort. This financial support is expressed in terms of the participation rate, which is defined as the fraction of the total advertising cost of one party's advertising effort that is shared by the other. Thus, manufacturer's participation rate for the retailer is the fraction of the cost of retailer's local advertising effort shared by the manufacturer. Similarly, the retailer's participation rate for the manufacturer is the fraction of the cost of manufacturer's national advertising effort shared by the retailer. The cost of advertising is expressed as the square of the advertising effort. This type of cost structure captures the marginally diminishing returns of advertising and is commonly used in the literature ([17] and [24]).

We denote the market share at time $t$ as $x(t)$. The following table lists the notation used in this paper. Here we take the manufacturer as Player 1 and the retailer as Player 2. So we will use subscripts $m$ and $r$ instead of 1 and 2 , respectively. Thus, $u_{1}, v_{1}, u_{2}, v_{2}$ will be replaced by $u_{m}, v_{m}, u_{r}, v_{r}$, respectively, in Sections 3-4.

$$
\begin{array}{cl}
m, r & \text { subscript used for manufacturer and retailer, respectively, } \\
x(t) \in[0,1] & \text { market share at time } t, \\
v_{m}(t) \in \mathcal{V}_{m}=[0, \infty) & \text { manufacturer's advertising effort rate at time } t, \\
v_{r}(t) \in \mathcal{V}_{r}=[0, \infty) & \text { retailer's advertising effort rate at time } t,
\end{array}
$$


$u_{m}(t) \in \mathcal{U}_{m}=[0,1]$ manufacturer's participation rate for retailer's advertising effort at time $t$, $u_{r}(t) \in \mathcal{U}_{r}=[0,1] \quad$ retailer's participation rate for manufacturer's advertising effort at time $t$, $a>0 \quad$ advertising effectiveness of retailer,

$b>0 \quad$ advertising effectiveness of manufacturer,

$\delta \geq 0 \quad$ market share decay parameter,

$\rho>0 \quad$ discount rate,

$R \geq 0 \quad$ gross margin of retailer,

$M \geq 0 \quad$ gross margin of manufacturer,

$V_{m} \quad$ value function of manufacturer,

$V_{r} \quad$ value function of retailer.

We model the sales-advertising dynamics as the following extension of the Sethi model, to incorporate multiple advertising decisions:

$$
\left\{\begin{array}{l}
\mathrm{d} x(t)=\left[\left(a v_{r}(t)+b v_{m}(t)+k \sqrt{v_{r}(t) v_{m}(t)}\right) \sqrt{1-x(t)}-\delta x(t)\right] d t+\sigma(x(t)) \mathrm{d} W(t), \\
x(0)=x_{0} \in[0,1]
\end{array}\right.
$$

where $k>0$ denotes the magnitude of synergy and $\sigma(x(t))$ is uniformly bounded with $\sigma(x(0))=\sigma(x(1))=0$. Under the model assumption, the market share $x(t)$ can be readily shown to be bounded in $[0,1]$.

The supply chain carries out its decisions in a Stackelberg framework. We assume that $m$ and $r$ announce their respective participation rates $u_{m}$ and $u_{r}$ first. Thus, the participation rates are the lead decisions. Given these, $m$ and $r$ decide on the national and local advertising rates, $v_{m}$ and $v_{r}$, respectively. That is, the advertising rates are the follow decisions. Thus, we have a special case of the mixed leadership differential game developed in Section 2. Accordingly, $m$ and $r$ play Nash to solve for $v_{m}$ and $v_{r}$ given $u_{m}$ and $u_{r}$. This produces optimal responses, which are then used by $m$ and $r$ in solving for $u_{m}$ and $u_{r}$, respectively, once again in the Nash differential game framework. The Stackelberg step is the use of the response function in the formulation of the Nash game for the lead decisions.

More specifically, the mixed-leadership game between the manufactuer and the retailer can be succinctly represented as follows:

Problem 3.1. The manufacturer chooses $\left(u_{m}, v_{m}\right) \in \mathcal{U}_{m} \times \mathcal{V}_{m}$ to maximize her accumulated 
revenue net of the cumulative advertising cost, i.e.,

$$
V_{m}(x)=\sup _{\left(u_{m}, v_{m}\right) \in \mathcal{U}_{m} \times \mathcal{V}_{m}} \mathbb{E}\left[\int_{0}^{\infty} \mathrm{e}^{-\rho t}\left(M x-u_{m} v_{r}^{2}-\left(1-u_{r}\right) v_{m}^{2}\right) \mathrm{d} t\right],
$$

while the retailer chooses $\left(u_{r}, v_{r}\right) \in \mathcal{U}_{r} \times \mathcal{V}_{r}$ to maximize her accumulated revenue net of the cumulative advertising cost, i.e.,

$$
V_{r}(x)=\sup _{\left(u_{r}, v_{r}\right) \in \mathcal{U}_{r} \times \mathcal{V}_{r}} \mathbb{E}\left[\int_{0}^{\infty} \mathrm{e}^{-\rho t}\left(R x-\left(1-u_{m}\right) v_{r}^{2}-u_{r} v_{m}^{2}\right) \mathrm{d} t\right],
$$

subject to the dynamics of the market share $x(t)$ in (24).

Under the model specifications in Problem 3.1, the Hamiltonians for $m$ and $r$ are as follows:

$$
\left\{\begin{aligned}
H_{m}\left(x, u_{m}, u_{r}, v_{m}, v_{r}, p_{m}\right)= & M x-u_{m} v_{r}^{2}-\left(1-u_{r}\right) v_{m}^{2} \\
& +p_{m}\left[\left(a v_{r}+b v_{m}+k \sqrt{v_{m} v_{r}}\right) \sqrt{1-x}-\delta x\right], \\
H_{r}\left(x, u_{m}, u_{r}, v_{m}, v_{r}, p_{r}\right)= & R x-\left(1-u_{m}\right) v_{r}^{2}-u_{r} v_{m}^{2} \\
& +p_{r}\left[\left(a v_{r}+b v_{m}+k \sqrt{v_{m} v_{r}}\right) \sqrt{1-x}-\delta x\right] .
\end{aligned}\right.
$$

The next theorem shows that the Problem 3.1 admits an analytical solution.

Theorem 3.1. The feedback Stackelberg-Nash equilibrium and the corresponding value functions to Problem 3.1 admit the following form:

$$
\begin{gathered}
\left\{\begin{array}{l}
v_{m}^{*}=\gamma_{m}^{2} \sqrt{1-x}, \\
v_{r}^{*}=\gamma_{r}^{2} \sqrt{1-x},
\end{array}\right. \\
\left\{\begin{array}{l}
u_{m}^{*}=\max \left(\hat{u}_{m}, 0\right), \quad \text { where } \quad\left(1-\hat{u}_{m}\right)=\frac{\beta_{m}\left(2 a \gamma_{r}+k \gamma_{m}\right)}{4 \gamma_{r}^{3}}, \\
u_{r}^{*}=\max \left(\hat{u}_{r}, 0\right), \quad \text { where } \quad\left(1-\hat{u}_{r}\right)=\frac{\beta_{r}\left(2 b \gamma_{m}+k \gamma_{r}\right)}{4 \gamma_{m}^{3}},
\end{array}\right. \\
\left\{\begin{array}{l}
V_{m}(x)=\alpha_{m}+\beta_{m} x, \\
V_{r}(x)=\alpha_{r}+\beta_{r} x,
\end{array}\right.
\end{gathered}
$$

where $\alpha_{r}, \alpha_{m}, \beta_{r}, \beta_{m}, \gamma_{r}$, and $\gamma_{m}$ are the solutions to the following coupled system of algrebraic 
equations:

$$
\left\{\begin{array}{l}
0=\left[4 \beta_{m}\left(a+\frac{k}{2} \frac{\gamma_{m}}{\gamma_{r}}\right)-8 \gamma_{r}^{2}\right]\left(2 b+\frac{3 k}{2} \frac{\gamma_{r}}{\gamma_{m}}\right)+2 a \beta_{r}\left(b+\frac{k}{2} \frac{\gamma_{r}}{\gamma_{m}}\right)+2 b \beta_{r}\left(a+\frac{k}{2} \frac{\gamma_{m}}{\gamma_{r}}\right) \\
0=\left[4 \beta_{r}\left(b+\frac{k}{2} \frac{\gamma_{r}}{\gamma_{m}}\right)-8 \gamma_{m}^{2}\right]\left(2 a+\frac{3 k}{2} \frac{\gamma_{m}}{\gamma_{r}}\right)+2 a \beta_{m}\left(b+\frac{k}{2} \frac{\gamma_{r}}{\gamma_{m}}\right)+2 b \beta_{m}\left(a+\frac{k}{2} \frac{\gamma_{m}}{\gamma_{r}}\right) \\
\rho \alpha_{m}=\beta_{m}\left(a \gamma_{r}^{2}+b \gamma_{m}^{2}+k \gamma_{r} \gamma_{m}\right)-\frac{\beta_{m} \gamma_{m}^{2}}{2}\left(b+\frac{k}{2} \frac{\gamma_{r}}{\gamma_{m}}\right)-\gamma_{r}^{4}+\frac{\beta_{r} \gamma_{r}^{2}}{2}\left(a+\frac{k}{2} \frac{\gamma_{m}}{\gamma_{r}}\right) \\
\rho \alpha_{r}=\beta_{r}\left(a \gamma_{r}^{2}+b \gamma_{m}^{2}+k \gamma_{r} \gamma_{m}\right)-\frac{\beta_{r} \gamma_{r}^{2}}{2}\left(a+\frac{k}{2} \frac{\gamma_{m}}{\gamma_{r}}\right)-\gamma_{m}^{4}+\frac{\beta_{m} \gamma_{m}^{2}}{2}\left(b+\frac{k}{2} \frac{\gamma_{r}}{\gamma_{m}}\right) \\
(\rho+\delta) \beta_{m}=M-\beta_{m}\left(a \gamma_{r}^{2}+b \gamma_{m}^{2}+k \gamma_{r} \gamma_{m}\right)+\frac{\beta_{m} \gamma_{m}^{2}}{2}\left(b+\frac{k}{2} \frac{\gamma_{r}}{\gamma_{m}}\right)+\gamma_{r}^{4}-\frac{\beta_{r} \gamma_{r}^{2}}{2}\left(a+\frac{k}{2} \frac{\gamma_{m}}{\gamma_{r}}\right) \\
(\rho+\delta) \beta_{r}=R-\beta_{r}\left(a \gamma_{r}^{2}+b \gamma_{m}^{2}+k \gamma_{r} \gamma_{m}\right)+\frac{\beta_{r} \gamma_{r}^{2}}{2}\left(a+\frac{k}{2} \frac{\gamma_{m}}{\gamma_{r}}\right)+\gamma_{m}^{4}-\frac{\beta_{m} \gamma_{m}^{2}}{2}\left(b+\frac{k}{2} \frac{\gamma_{r}}{\gamma_{m}}\right)
\end{array}\right.
$$

To prove Theorem 3.1, we shall first need Propositions 3.2, Corollary 3.3, and Proposition 3.4 .

Proposition 3.2. Given $u_{m}, u_{r} \in[0,1]$ and $p_{m}, p_{r}>0$, the solution pair $\left(\Gamma_{m}^{v}, \Gamma_{r}^{v}\right)$ of $(6)$ admits the following form:

(i) If there is at least one $u_{i}=1$, for $i=m, r$, then there is no solution $\left(v_{m}, v_{r}\right)$ satisfying (6) simultaneously for $x \neq 1$;

(ii) If $u_{m} \neq 1, u_{r} \neq 1$, then

$$
\left\{\begin{array}{l}
\Gamma_{m}^{v}\left(x, u_{m}, u_{r}, p_{m}, p_{r}\right)=\frac{p_{m}}{2\left(1-u_{r}\right)}\left(b+\frac{k}{2} y\right) \sqrt{1-x} \\
\Gamma_{r}^{v}\left(x, u_{m}, u_{r}, p_{m}, p_{r}\right)=\frac{p_{r}}{2\left(1-u_{m}\right)}\left(a+\frac{k}{2 y}\right) \sqrt{1-x}
\end{array}\right.
$$

where $y \triangleq \frac{v_{2}}{v_{1}}$ is the unique solution to the following equation

$$
0=\frac{k p_{m}}{2 p_{r}} y^{4}+\frac{b p_{m}}{p_{r}} y^{3}-a \frac{\left(1-u_{r}\right)}{\left(1-u_{m}\right)} y-\frac{k\left(1-u_{r}\right)}{2\left(1-u_{m}\right)} .
$$

Proof. Given $u_{m}, u_{r} \in[0,1]$ and $p_{m}, p_{r}>0$, the first-order condition of $H_{m}$ (resp., $\left.H_{r}\right)$ in (27) with respect to $v_{m}$ (resp., $v_{r}$ ) gives

$$
\begin{aligned}
2 v_{m}\left(1-u_{r}\right) & =p_{m}\left(b+\frac{k}{2} \sqrt{\frac{v_{r}}{v_{m}}}\right) \sqrt{1-x} \\
2 v_{r}\left(1-u_{m}\right) & =p_{r}\left(a+\frac{k}{2} \sqrt{\frac{v_{m}}{v_{r}}}\right) \sqrt{1-x}
\end{aligned}
$$

We now analyze the solution pair $\left(v_{m}, v_{r}\right)$ of (34)-(35) under the following cases of $u_{m}, u_{r}$ : 
(i) (a) $\left(u_{m}=1, u_{r}=1\right)$

In this case, there is no solution $\left(v_{m}, v_{r}\right)$ when $\left(u_{m}, u_{r}\right)=(1,1)$, for $x \neq 1$. Indeed, when $\left(u_{m}, u_{r}\right)=(1,1)$, the left-hand sides of (34) and (35) are zero. Since $p_{m}, p_{r}, a, b, k>0$, it follows readily that the right-hand sides of (34) and (35) cannot be zero simultaneously unless $x=1$.

(b) $\left(u_{m} \neq 1, u_{r}=1\right)$

When $u_{m} \neq 1, u_{r}=1$, (34) yields $\sqrt{\frac{v_{r}}{v_{m}}}=-\frac{2 b}{k}$ or $v_{r}=\frac{4 b^{2}}{k^{2}} v_{m}$. Hence, (35) now becomes

$$
v_{m}=\frac{k^{2}}{8 b^{2}} \frac{p_{r}}{\left(1-u_{m}\right)}\left(a-\frac{k^{2}}{4 b}\right) \sqrt{1-x},
$$

which implies that

$$
v_{r}=\frac{p_{r}}{2\left(1-u_{m}\right)}\left(a-\frac{k^{2}}{4 b}\right) \sqrt{1-x} .
$$

Note: Substituting in the above expressions for $v_{m}, v_{r}$ yields $\frac{v_{r}}{v_{m}}=\frac{4 b^{2}}{k^{2}}$. Since $u_{r}=1,(34)$ becomes $0=p_{m}(b+b) \sqrt{1-x}$, which can only be true when $x=1$, as Case (a).

(c) $\left(u_{m}=1, u_{r} \neq 1\right)$

Following the analogous arguments in Case (b), it also follows readily that

$$
\left\{\begin{array}{l}
v_{r}=\frac{k^{2}}{8 a^{2}} \frac{p_{m}}{\left(1-u_{r}\right)}\left(b-\frac{k^{2}}{4 a}\right) \sqrt{1-x}, \\
v_{m}=\frac{p_{m}}{2\left(1-u_{r}\right)}\left(b-\frac{k^{2}}{4 a}\right) \sqrt{1-x}
\end{array}\right.
$$

implying that $\frac{v_{m}}{v_{r}}=\frac{4 a^{2}}{k^{2}}$. Since $u_{m}=1,(35)$ becomes $0=p_{r}(a+a) \sqrt{1-x}$, which can only be true when $x=1$, as Case (a).

(ii) $\left(u_{m} \neq 1, u_{r} \neq 1\right)$

We first show that $\left(v_{m}, v_{r}\right)$ to $(34)-(35)$ are bounded away from zero. Indeed, when $v_{m} \rightarrow 0$, the left-hand side of $(34)$ converges to 0 for $u_{r} \neq 1$ on $[0,1]$, whereas the righthand side of (34) converges to $\infty$. On the other hand, when $v_{r} \rightarrow 0$, the left-hand side of $(35)$ converges to 0 for $u_{m} \neq 1$, whereas the right-hand side of (35) converges to $\infty$. Now dividing (34) by (35) gives

$$
\frac{v_{m}\left(1-u_{r}\right)}{v_{r}\left(1-u_{m}\right)}=\frac{p_{m}}{p_{r}} \frac{\left(b+\frac{k}{2} \sqrt{\frac{v_{r}}{v_{m}}}\right)}{\left(a+\frac{k}{2} \sqrt{\frac{v_{m}}{v_{r}}}\right)} .
$$


Denote $y \triangleq \sqrt{\frac{v_{r}}{v_{m}}}$. Rearranging (36) yields

$$
0=\frac{k p_{m}}{2 p_{r}} y^{4}+\frac{b p_{m}}{p_{r}} y^{3}-a \frac{\left(1-u_{r}\right)}{\left(1-u_{m}\right)} y-\frac{k\left(1-u_{r}\right)}{2\left(1-u_{m}\right)}
$$

Define

$$
\zeta(y) \triangleq \frac{k p_{m}}{2 p_{r}} y^{4}+\frac{b p_{m}}{p_{r}} y^{3}-a \frac{\left(1-u_{r}\right)}{\left(1-u_{m}\right)} y-\frac{k\left(1-u_{r}\right)}{2\left(1-u_{m}\right)}
$$

Since

$$
\lim _{y \rightarrow 0} \zeta(y)=-\frac{k\left(1-u_{r}\right)}{2\left(1-u_{m}\right)}<0, \quad \lim _{y \rightarrow \infty} \zeta(y)=\infty
$$

implying that there exists at least one $y^{*} \in(0, \infty)$ such that $\zeta\left(y^{*}\right)=0$.

To show that $y^{*}>0$ satisfying $\zeta\left(y^{*}\right)=0$ is unique, observe that

$$
\begin{aligned}
\left.\zeta^{\prime}(y)\right|_{\zeta(y)=0} & =\frac{2 k p_{m}}{p_{r}} y^{3}+\frac{3 b p_{m}}{p_{r}} y^{2}-a \frac{\left(1-u_{r}\right)}{\left(1-u_{m}\right)} \\
& =\frac{k p_{m}}{p_{r}} y^{3}+\frac{2 b p_{m}}{p_{r}} y^{2}+\frac{1}{y}\left(\frac{k p_{m}}{2 p_{r}} y^{4}+\frac{b p_{m}}{p_{r}} y^{3}-a \frac{\left(1-u_{r}\right)}{\left(1-u_{m}\right)} y\right) \\
& =\frac{k p_{m}}{2 p_{r}} y^{3}+\frac{2 b p_{m}}{p_{r}} y^{2}+\left(\frac{1}{y}\right) \frac{k\left(1-u_{r}\right)}{2\left(1-u_{m}\right)}>0,
\end{aligned}
$$

where the last equality follows from $\zeta(y)=0$. Therefore, we have a unique $y^{*}$ satisfying $\zeta\left(y^{*}\right)=0$.

Corollary 3.3. Given $u_{m}, u_{r}, p_{m}, p_{r}>0$, the partial derivatives $\frac{\partial v_{1}}{\partial u_{2}}, \frac{\partial v_{1}}{\partial u_{2}}>0$ and admit the following explicit forms, for $u_{m}, u_{r} \neq 1$,

$$
\begin{aligned}
\frac{\partial \Gamma_{m}^{v}\left(x, u_{m}, u_{r}, p_{m}, p_{r}\right)}{\partial u_{r}} & =\frac{\frac{2 v_{m}}{\left(1-u_{r}\right)}\left(b+\frac{k}{2} \sqrt{\frac{v_{r}}{v_{m}}}\right)\left(2 a+\frac{3 k}{2} \sqrt{\frac{v_{m}}{v_{r}}}\right)}{\Delta\left(v_{m}, v_{r}\right)}, \\
\frac{\partial \Gamma_{r}^{v}\left(x, u_{m}, u_{r}, p_{m}, p_{r}\right)}{\partial u_{m}} & =\frac{\frac{2 v_{r}}{\left(1-u_{m}\right)}\left(a+\frac{k}{2} \sqrt{\frac{v_{m}}{v_{r}}}\right)\left(2 b+\frac{3 k}{2} \sqrt{\frac{v_{r}}{v_{m}}}\right)}{\Delta\left(v_{m}, v_{r}\right)},
\end{aligned}
$$

where

$$
\Delta\left(v_{m}, v_{r}\right) \triangleq 4\left(a+\frac{k}{2} \sqrt{\frac{v_{m}}{v_{r}}}\right)\left(b+\frac{k}{2} \sqrt{\frac{v_{r}}{v_{m}}}\right)+k \sqrt{\frac{v_{m}}{v_{r}}}\left(b+\frac{k}{2} \sqrt{\frac{v_{r}}{v_{m}}}\right)+k \sqrt{\frac{v_{r}}{v_{m}}}\left(a+\frac{k}{2} \sqrt{\frac{v_{m}}{v_{r}}}\right) .
$$


Proof. Differentiating (34) and (35) with respect to $u_{m}$ yields

$$
\left\{\begin{array}{l}
2\left(1-u_{r}\right) \frac{\partial v_{m}}{\partial u_{m}}=p_{m}\left(\frac{k}{4} v_{r}^{-\frac{1}{2}} v_{m}^{-\frac{1}{2}} \frac{\partial v_{r}}{\partial u_{m}}-\frac{k}{4} v_{r}^{\frac{1}{2}} v_{m}^{-\frac{3}{2}} \frac{\partial v_{m}}{\partial u_{m}}\right) \sqrt{1-x} \\
2\left(1-u_{m}\right) \frac{\partial v_{r}}{\partial u_{m}}-2 v_{r}=p_{r}\left(\frac{k}{4} v_{m}^{-\frac{1}{2}} v_{r}^{-\frac{1}{2}} \frac{\partial v_{m}}{\partial u_{m}}-\frac{k}{4} v_{m}^{\frac{1}{2}} v_{r}^{-\frac{3}{2}} \frac{\partial v_{r}}{\partial u_{m}}\right) \sqrt{1-x}
\end{array}\right.
$$

or, in matrix form, we have

$$
\left(\begin{array}{c}
0 \\
2 v_{r}
\end{array}\right)=\mathbf{A}\left(\begin{array}{c}
\frac{\partial v_{m}}{\partial u_{m}} \\
\frac{\partial v_{r}}{\partial u_{m}}
\end{array}\right)
$$

where

$$
\mathbf{A} \triangleq\left(\begin{array}{cc}
2\left(1-u_{r}\right)+\frac{k}{4} p_{m} v_{r}^{\frac{1}{2}} v_{m}^{-\frac{3}{2}} \sqrt{1-x} & -\frac{k}{4} p_{m} v_{r}^{-\frac{1}{2}} v_{m}^{-\frac{1}{2}} \sqrt{1-x} \\
-\frac{k}{4} p_{r} v_{m}^{-\frac{1}{2}} v_{r}^{-\frac{1}{2}} \sqrt{1-x} & 2\left(1-u_{m}\right)+\frac{k}{4} p_{r} v_{m}^{\frac{1}{2}} v_{r}^{-\frac{3}{2}} \sqrt{1-x}
\end{array}\right)
$$

Since

$$
\begin{aligned}
\operatorname{det}(\mathbf{A}) & =4\left(1-u_{m}\right)\left(1-u_{r}\right)+\frac{\left(1-u_{r}\right) k}{2} v_{m}^{\frac{1}{2}} v_{r}^{-\frac{3}{2}} p_{r} \sqrt{1-x}+\frac{\left(1-u_{m}\right) k}{2} v_{r}^{\frac{1}{2}} v_{m}^{-\frac{3}{2}} p_{m} \sqrt{1-x} \\
& =4\left(1-u_{m}\right)\left(1-u_{r}\right)+\frac{\left(1-u_{r}\right)\left(1-u_{m}\right) k \sqrt{\frac{v_{m}}{v_{r}}}}{\left(a+\frac{k}{2} \sqrt{\frac{v_{m}}{v_{r}}}\right)}+\frac{\left(1-u_{r}\right)\left(1-u_{m}\right) k \sqrt{\frac{v_{r}}{v_{m}}}}{\left(b+\frac{k}{2} \sqrt{\frac{v_{r}}{v_{m}}}\right)} \\
& =\left(1-u_{m}\right)\left(1-u_{r}\right) \frac{\Delta\left(v_{m}, v_{r}\right)}{\left(a+\frac{k}{2} \sqrt{\frac{v_{m}}{v_{r}}}\right)\left(b+\frac{k}{2} \sqrt{\frac{v_{r}}{v_{m}}}\right)}>0
\end{aligned}
$$

where the second equality follows from (34) and (35) and the third follows from (40).

It follows from (41) that

$$
\left(\begin{array}{c}
\frac{\partial v_{m}}{\partial u_{m}} \\
\frac{\partial v_{r}}{\partial u_{m}}
\end{array}\right)=\frac{\mathbf{A}^{-1}}{\operatorname{det}(\mathbf{A})}\left(\begin{array}{c}
0 \\
2 v_{r}
\end{array}\right)
$$

where $\mathbf{A}^{-1}=\left(\begin{array}{cc}2\left(1-u_{m}\right)+\frac{k}{4} p_{r} v_{m}^{\frac{1}{2}} v_{r}^{-\frac{3}{2}} \sqrt{1-x} & \frac{k}{4} p_{m} v_{r}^{-\frac{1}{2}} v_{m}^{-\frac{1}{2}} \sqrt{1-x} \\ \frac{k}{4} p_{r} v_{m}^{-\frac{1}{2}} v_{r}^{-\frac{1}{2}} \sqrt{1-x} & 2\left(1-u_{r}\right)+\frac{k}{4} p_{m} v_{r}^{\frac{1}{2}} v_{m}^{-\frac{3}{2}} \sqrt{1-x}\end{array}\right)$ 
This implies that

$$
\begin{aligned}
\frac{\partial v_{r}}{\partial u_{m}} & =\frac{2 v_{r}}{\operatorname{det}(\mathbf{A})}\left[2\left(1-u_{r}\right)+\frac{k}{4} v_{r}^{\frac{1}{2}} v_{m}^{-\frac{3}{2}} p_{m} \sqrt{1-x}\right] \\
& =\frac{2 v_{r}}{\operatorname{det}(\mathbf{A})}\left[2\left(1-u_{r}\right)+\frac{\frac{k}{2} \sqrt{\frac{v_{r}}{v_{m}}}\left(1-u_{r}\right)}{\left.\left(b+\frac{k}{2} \sqrt{\frac{v_{r}}{v_{m}}}\right)\right]}\right. \\
& =\frac{\frac{2 v_{r}}{\left(1-u_{m}\right)}\left(a+\frac{k}{2} \sqrt{\frac{v_{m}}{v_{r}}}\right)\left(2 b+\frac{3 k}{2} \sqrt{\frac{v_{r}}{v_{m}}}\right)}{\Delta\left(v_{m}, v_{r}\right)},
\end{aligned}
$$

where the second equality follows from (34), the third equality follows from (42). In addition, it is now readily seen from $(40)$ that $\frac{\partial v_{r}}{\partial u_{m}}>0$.

By the exact arguments, we obtain

$$
\begin{aligned}
\frac{\partial v_{m}}{\partial u_{r}} & =\frac{2 v_{m}}{\operatorname{det}(\mathbf{A})}\left[2\left(1-u_{m}\right)+\frac{k}{4} v_{m}^{\frac{1}{2}} v_{r}^{-\frac{3}{2}} p_{r} \sqrt{1-x}\right] \\
& =\frac{2 v_{m}}{\operatorname{det}(\mathbf{A})}\left[2\left(1-u_{m}\right)+\frac{\frac{k}{2} \sqrt{\frac{v_{m}}{v_{r}}}\left(1-u_{m}\right)}{\left.\left(a+\frac{k}{2} \sqrt{\frac{v_{m}}{v_{r}}}\right)\right]}\right. \\
& =\frac{\frac{2 v_{m}}{\left(1-u_{r}\right)}\left(b+\frac{k}{2} \sqrt{\frac{v_{r}}{v_{m}}}\right)\left(2 a+\frac{3 k}{2} \sqrt{\frac{v_{m}}{v_{r}}}\right)}{\Delta\left(v_{m}, v_{r}\right)},
\end{aligned}
$$

where the second equality follows from (35), the third equality follows from (42). Finally, it is now readily seen from (40) that $\frac{\partial v_{m}}{\partial u_{r}}>0$. This concludes the proof.

Proposition 3.4. Given $p_{m}, p_{r}>0$, the solution pair $\left(\Gamma_{m}^{\mu}, \Gamma_{r}^{\mu}\right)$ of (7) admits the following explicit form:

$$
\left\{\begin{array}{l}
\Gamma_{m}^{\mu}\left(x, p_{m}, p_{r}\right)=\min \left(1-\bar{u}_{m}, 0\right) \\
\Gamma_{r}^{\mu}\left(x, p_{m}, p_{r}\right)=\min \left(1-\bar{u}_{r}, 0\right)
\end{array}\right.
$$

where

$$
\begin{aligned}
& \bar{u}_{m}=\frac{4\left(a+\frac{k}{2} \sqrt{\frac{v_{m}}{v_{r}}}\right)\left(2 b+\frac{3 k}{2} \sqrt{\frac{v_{r}}{v_{m}}}\right)}{\frac{4 p_{m}}{p_{r}}\left(a+\frac{k}{2} \sqrt{\frac{v_{m}}{v_{r}}}\right)\left(2 b+\frac{3 k}{2} \sqrt{\frac{v_{r}}{v_{m}}}\right)+4 a b+a k \sqrt{\frac{v_{r}}{v_{m}}}+b k \sqrt{\frac{v_{m}}{v_{r}}}}, \\
& \bar{u}_{r}=\frac{4\left(b+\frac{k}{2} \sqrt{\frac{v_{r}}{v_{m}}}\right)\left(2 a+\frac{3 k}{2} \sqrt{\frac{v_{m}}{v_{r}}}\right)}{\frac{4 p_{r}}{p_{m}}\left(b+\frac{k}{2} \sqrt{\frac{v_{r}}{v_{m}}}\right)\left(2 a+\frac{3 k}{2} \sqrt{\frac{v_{m}}{v_{r}}}\right)+4 a b+a k \sqrt{\frac{v_{r}}{v_{m}}}+b k \sqrt{\frac{v_{m}}{v_{r}}}}
\end{aligned}
$$


Proof. The first-order condition of $H_{m}\left(\right.$ resp. $\left.H_{r}\right)$ in $(27)$ with respect to $u_{m}$ (resp., $u_{r}$ ) gives

$$
\left\{\begin{aligned}
0=-v_{r}^{2}+ & \frac{\partial v_{r}}{\partial u_{m}}\left[-2 u_{m} v_{r}+p_{m}\left(a+\frac{k}{2} \sqrt{\frac{v_{m}}{v_{r}}}\right) \sqrt{1-x}\right] \\
+ & \frac{\partial v_{m}}{\partial u_{m}}\left[-2\left(1-u_{r}\right) v_{m}+p_{m}\left(b+\frac{k}{2} \sqrt{\frac{v_{r}}{v_{m}}}\right) \sqrt{1-x}\right] \\
0=-v_{m}^{2}+ & \frac{\partial v_{m}}{\partial u_{r}}\left[-2 u_{r} v_{m}+p_{r}\left(b+\frac{k}{2} \sqrt{\frac{v_{r}}{v_{m}}}\right) \sqrt{1-x}\right] \\
+ & \frac{\partial v_{r}}{\partial u_{r}}\left[-2\left(1-u_{m}\right) v_{r}+p_{r}\left(a+\frac{k}{2} \sqrt{\frac{v_{m}}{v_{r}}}\right) \sqrt{1-x}\right]
\end{aligned}\right.
$$

In view of (34) and (35), (46) can be simplified as follows:

$$
\left\{\begin{array}{l}
0=-v_{r}^{2}+\frac{\partial v_{r}}{\partial u_{m}}\left[-2 u_{m} v_{r}+2 \frac{p_{m}}{p_{r}} v_{r}\left(1-u_{m}\right)\right] \\
0=-v_{m}^{2}+\frac{\partial v_{m}}{\partial u_{r}}\left[-2 u_{r} v_{m}+2 \frac{p_{r}}{p_{m}} v_{m}\left(1-u_{r}\right)\right]
\end{array}\right.
$$

In view of $\frac{\partial v_{r}}{\partial u_{m}}$ in $(39)$, the first equation in (47) becomes

$$
\begin{aligned}
0 & =-v_{r}^{2}+2 v_{r} \frac{\partial v_{r}}{\partial u_{m}}\left[\left(1-u_{m}\right)\left(1+\frac{p_{m}}{p_{r}}\right)-1\right] \\
& =-v_{r}^{2}+\frac{4 v_{r}^{2}}{\left(1-u_{m}\right)}\left[\left(1-u_{m}\right)\left(1+\frac{p_{m}}{p_{r}}\right)-1\right]\left[\frac{\left(a+\frac{k}{2} \sqrt{\frac{v_{m}}{v_{r}}}\right)\left(2 b+\frac{3 k}{2} \sqrt{\frac{v_{r}}{v_{m}}}\right)}{\Delta\left(v_{m}, v_{r}\right)}\right] \\
& =-\left(1-u_{m}\right) \Delta\left(v_{m}, v_{r}\right)+4\left[\left(1-u_{m}\right)\left(1+\frac{p_{m}}{p_{r}}\right)-1\right]\left(a+\frac{k}{2} \sqrt{\frac{v_{m}}{v_{r}}}\right)\left(2 b+\frac{3 k}{2} \sqrt{\frac{v_{r}}{v_{m}}}\right),
\end{aligned}
$$

where the second equality follows from (39) in Corollary 3.3. Hence, we have

$$
\begin{aligned}
\left(1-u_{m}\right) & =\frac{4\left(a+\frac{k}{2} \sqrt{\frac{v_{m}}{v_{r}}}\right)\left(2 b+\frac{3 k}{2} \sqrt{\frac{v_{r}}{v_{m}}}\right)}{4\left(1+\frac{p_{m}}{p_{r}}\right)\left(a+\frac{k}{2} \sqrt{\frac{v_{m}}{v_{r}}}\right)\left(2 b+\frac{3 k}{2} \sqrt{\frac{v_{r}}{v_{m}}}\right)-\Delta\left(v_{m}, v_{r}\right)} \\
& =\frac{4\left(a+\frac{k}{2} \sqrt{\frac{v_{m}}{v_{r}}}\right)\left(2 b+\frac{3 k}{2} \sqrt{\frac{v_{r}}{v_{m}}}\right)}{\frac{4 p_{m}}{p_{r}}\left(a+\frac{k}{2} \sqrt{\frac{v_{m}}{v_{r}}}\right)\left(2 b+\frac{3 k}{2} \sqrt{\frac{v_{r}}{v_{m}}}\right)+4 a b+a k \sqrt{\frac{v_{r}}{v_{m}}}+b k \sqrt{\frac{v_{m}}{v_{r}}}}>0,
\end{aligned}
$$

where the second equality follows from (40). Exact arguments show that

$$
\begin{aligned}
\left(1-u_{r}\right) & =\frac{4\left(b+\frac{k}{2} \sqrt{\frac{v_{r}}{v_{m}}}\right)\left(2 a+\frac{3 k}{2} \sqrt{\frac{v_{m}}{v_{r}}}\right)}{4\left(1+\frac{p_{r}}{p_{m}}\right)\left(b+\frac{k}{2} \sqrt{\frac{v_{r}}{v_{m}}}\right)\left(2 a+\frac{3 k}{2} \sqrt{\frac{v_{m}}{v_{r}}}\right)-\Delta\left(v_{m}, v_{r}\right)} \\
& =\frac{4\left(b+\frac{k}{2} \sqrt{\frac{v_{r}}{v_{m}}}\right)\left(2 a+\frac{3 k}{2} \sqrt{\frac{v_{m}}{v_{r}}}\right)}{\frac{4 p_{r}}{p_{m}}\left(b+\frac{k}{2} \sqrt{\frac{v_{r}}{v_{m}}}\right)\left(2 a+\frac{3 k}{2} \sqrt{\frac{v_{m}}{v_{r}}}\right)+4 a b+a k \sqrt{\frac{v_{r}}{v_{m}}}+b k \sqrt{\frac{v_{m}}{v_{r}}}}>0 .
\end{aligned}
$$


This concludes the proof.

Proof of Theorem 3.1:

In view of Theorem 2.1, it suffices to show that the value functions $V_{m}, V_{r}$ in (30) solve the following ODE system:

$$
\left\{\begin{array}{l}
\rho V_{m}=M x-u_{m}^{*} v_{r}^{* 2}-\left(1-u_{r}^{*}\right) v_{m}^{* 2}+V_{m}^{\prime}\left[\left(a v_{r}^{*}+b v_{m}^{*}+k \sqrt{v_{r}^{*} v_{m}^{*}}\right) \sqrt{1-x}-\delta x\right]+\frac{V_{m}^{\prime \prime} \sigma(x)^{2}}{2}, \\
\rho V_{r}=R x-\left(1-u_{m}^{*}\right) v_{r}^{* 2}-u_{r} v_{m}^{* 2}+V_{r}^{\prime}\left[\left(a v_{r}^{*}+b v_{m}^{*}+k \sqrt{v_{r}^{*} v_{m}^{*}}\right) \sqrt{1-x}-\delta x\right]+\frac{V_{r}^{\prime \prime} \sigma(x)^{2}}{2},
\end{array}\right.
$$

where the solution pair $\left(v_{m}^{*}, v_{r}^{*}\right)$ of $(6)$ admits the form in (28) and the solution pair $\left(u_{m}^{*}, u_{r}^{*}\right)$ of (7) admits the form in (29) .

Indeed, in terms of the assumed forms of $V_{i}$ in (30) and $v_{i}$ in (28), for $i=r, m,(34)-(35)$ yield

$$
\left\{\begin{array}{l}
\left(1-u_{m}\right)=\frac{\beta_{r}}{2 \gamma_{r}^{2}}\left(a+\frac{k}{2} \frac{\gamma_{m}}{\gamma_{r}}\right) \\
\left(1-u_{r}\right)=\frac{\beta_{m}}{2 \gamma_{m}^{2}}\left(b+\frac{k}{2} \frac{\gamma_{r}}{\gamma_{m}}\right)
\end{array}\right.
$$

yielding (29). The desired expressions for $u_{r}^{*}$ and $u_{m}^{*}$ readily follow by recalling that $u_{r}^{*}$ and $u_{m}^{*}$ must be in $[0,1]$.

On the other hand, $\left(1-u_{r}\right)$ and $\left(1-u_{m}\right)$ in Proposition 3.4 now become

$$
\left\{\begin{array}{l}
\left(1-u_{m}\right)=\frac{4\left(a+\frac{k}{2} \frac{\gamma_{m}}{\gamma_{r}}\right)\left(2 b+\frac{3 k}{2} \frac{\gamma_{r}}{\gamma_{m}}\right)}{\frac{4 \beta_{m}}{\beta_{r}}\left(a+\frac{k}{2} \frac{\gamma_{m}}{\gamma_{r}}\right)\left(2 b+\frac{3 k}{2} \frac{\gamma_{r}}{v \gamma_{m}}\right)+4 a b+a k \frac{\gamma_{r}}{\gamma_{m}}+b k \frac{\gamma_{m}}{\gamma_{r}}}, \\
\left(1-u_{r}\right)=\frac{4\left(b+\frac{k}{2} \frac{\gamma_{r}}{\gamma_{m}}\right)\left(2 a+\frac{3 k}{2} \frac{\gamma_{m}}{\gamma_{r}}\right)}{\frac{4 \beta_{r}}{\beta_{m}}\left(b+\frac{k}{2} \frac{\gamma_{r}}{\gamma_{m}}\right)\left(2 a+\frac{3 k}{2} \frac{\gamma_{m}}{\gamma_{r}}\right)+4 a b+a k \frac{\gamma_{r}}{\gamma_{m}}+b k \frac{\gamma_{m}}{\gamma_{r}}} .
\end{array}\right.
$$

Equating (49) and (50) yields the first two equations in (31).

To determine the constants $\alpha_{m}, \alpha_{r}, \beta_{m}, \beta_{r}$, substituting the assumed forms of $V_{i}$ and $v_{i}$, together with $\left(1-u_{i}\right)$ in $(49)$, for $i=r, m$, into the ODE system in (48) and matching coefficients yields the remaining four equations in (31). This concludes the proof.

\section{Numerical Analysis}

Since it is quite difficult to obtain an explicit solution of the system of equations (31) in the general case, we performed a numerical analysis to get key insights into the dependence of the decision variables on the various model parameters. We numerically solved the above set of equations using Mathematica for a variety of parameter sets and obtained similar results. In this section we present some sample results highlighting the insights obtained. For this purpose, we define $C_{m}=u_{m} \gamma_{r}{ }^{4}+\left(1-u_{r}\right) \gamma_{m}{ }^{4}, C_{r}=u_{r} \gamma_{m}{ }^{4}+\left(1-u_{m}\right) \gamma_{r}{ }^{4}$, and 
$\Delta_{m-r}=u_{m} \gamma_{r}{ }^{4}-u_{r} \gamma_{m}{ }^{4}$. The discussion below highlights our findings on the impact of model parameters on the following: participation rates $\left(u_{m}, u_{r}\right)$, manufacturer's and retailer's total advertising expenses (represented by $C_{m}$, and $C_{r}$, respectively), total expenses on national and local advertising (represented by $\gamma_{m}{ }^{4}$, and $\gamma_{r}{ }^{4}$, respectively), and net transfer of money from the manufacturer to the retailer (represented by $\Delta_{m-r}$ ). We also investigate the impact of various parameters on the value functions of the two parties. Figures (5)-(23) show value functions of the retailer and the manufacturer for two different values of the initial market share, i.e., $x_{0}=0.1$ and $x_{0}=0.6$. All the figures corresponding to the numerical analysis are presented together following a brief discussion of results.

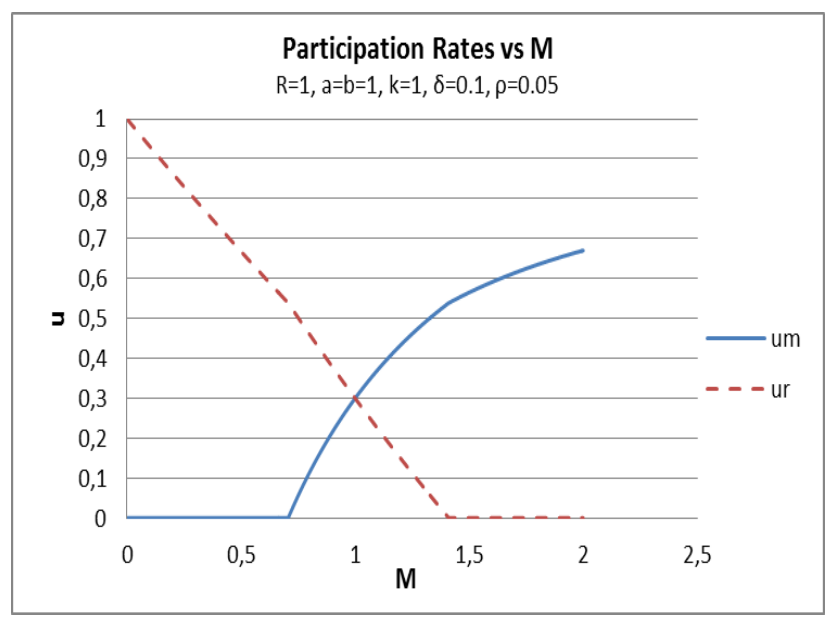

Figure 1: Participation Rates vs M

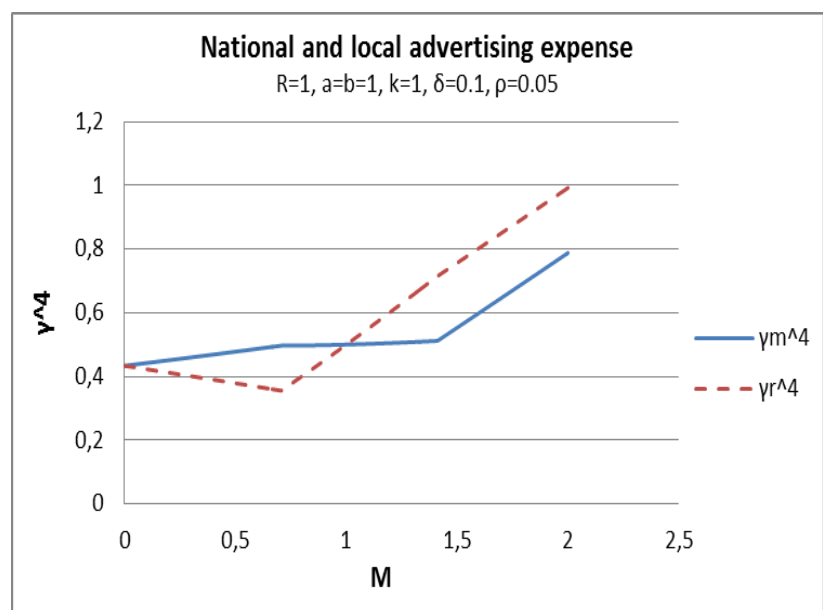

Figure 3: National \& local ad. expenses vs M

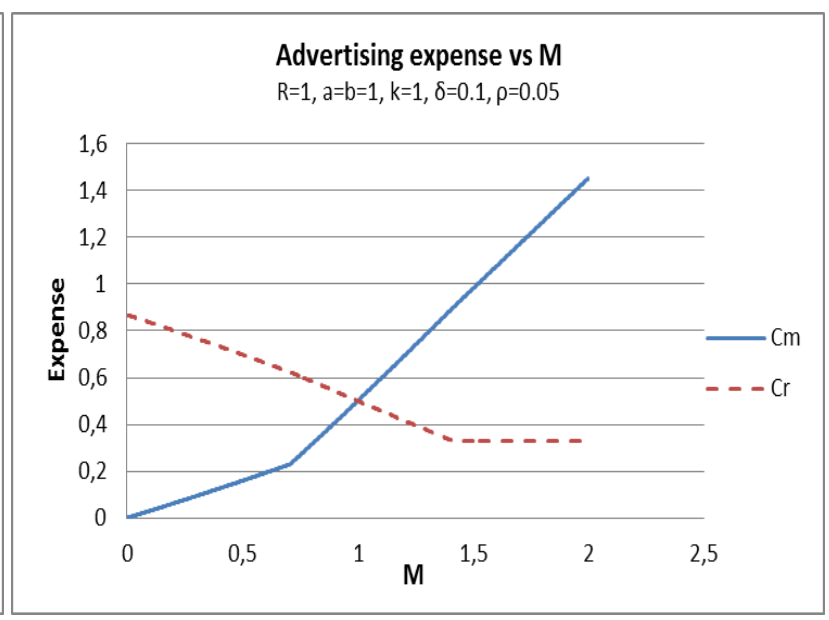

Figure 2: Mfg and ret's total ad. expenses vs M

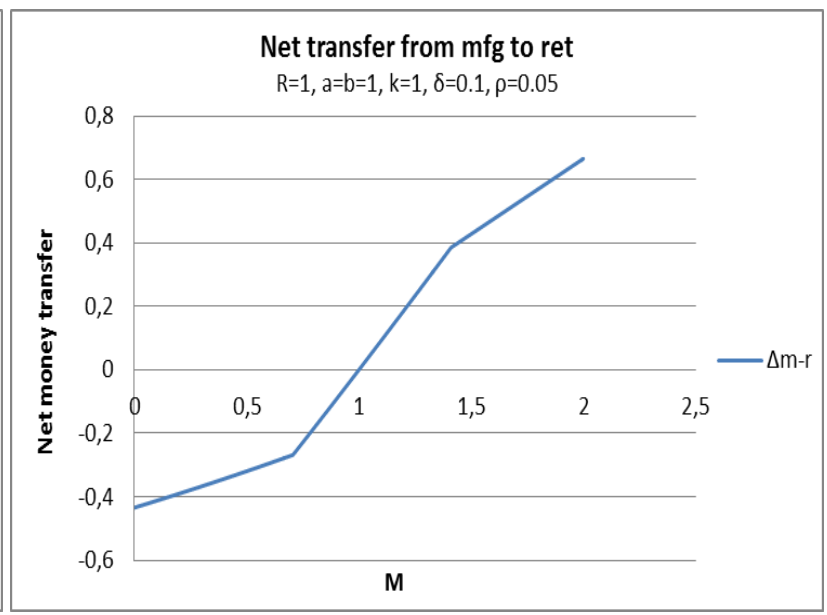

Figure 4: Net transfer from mfg to ret vs M

- Impact of margins (Figures 1-5): As the manufacturer's margin $M$ increases, the manufacturer has a higher incentive to advertise more. Thus, the manufacturer's support 


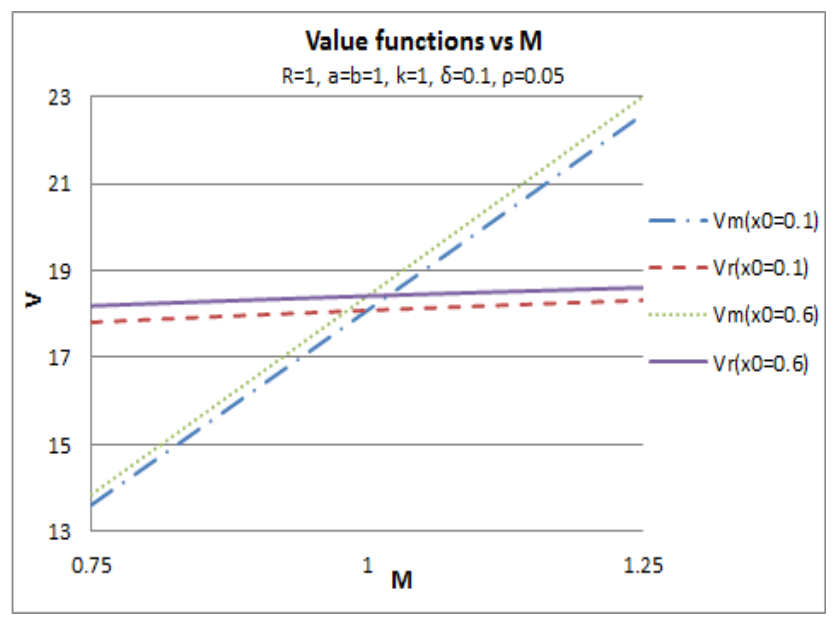

Figure 5: Value functions vs M

to the retailer increases whereas that of the retailer decreases. Also, the manufacturer's total expense in advertising increases whereas that of the retailer decreases. The national advertising is higher than the local when $M<R$ and vice versa. Also, as $M$ increases, the net money transfer from manufacturer to the retailer increases. This indicates the manufacturer's increasing incentive to the retailer to advertise more as greater advertising is more beneficial to the manufacturer than the retailer. Figure 5 shows that the value function of the manufacturer increases sharply as his margin $M$ increases, whereas the impact on the retailer is relatively much less prominent. Furthermore, the profits of both parties increase marginally as the initial market share increases. Above results are symmetric in nature when the retailer's margin $R$ changes instead.

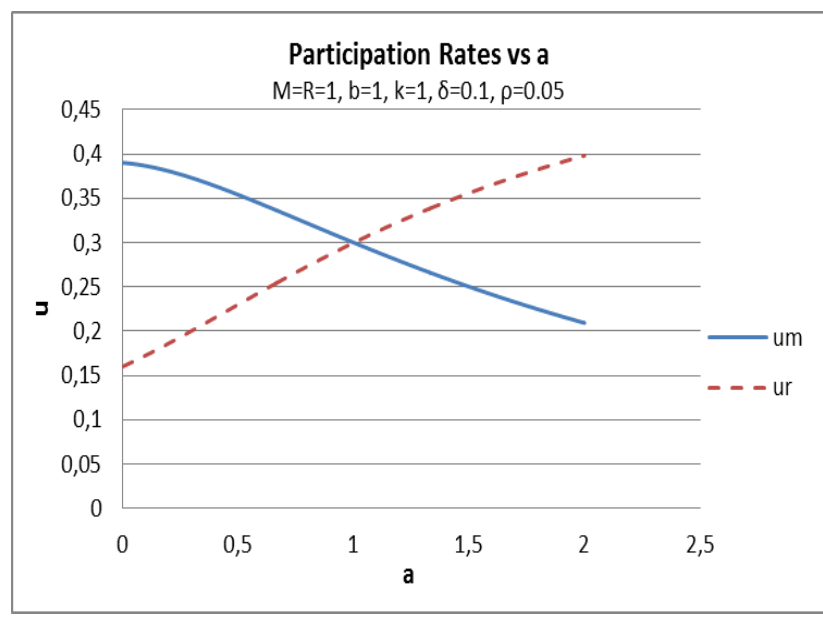

Figure 6: Participation Rates vs a

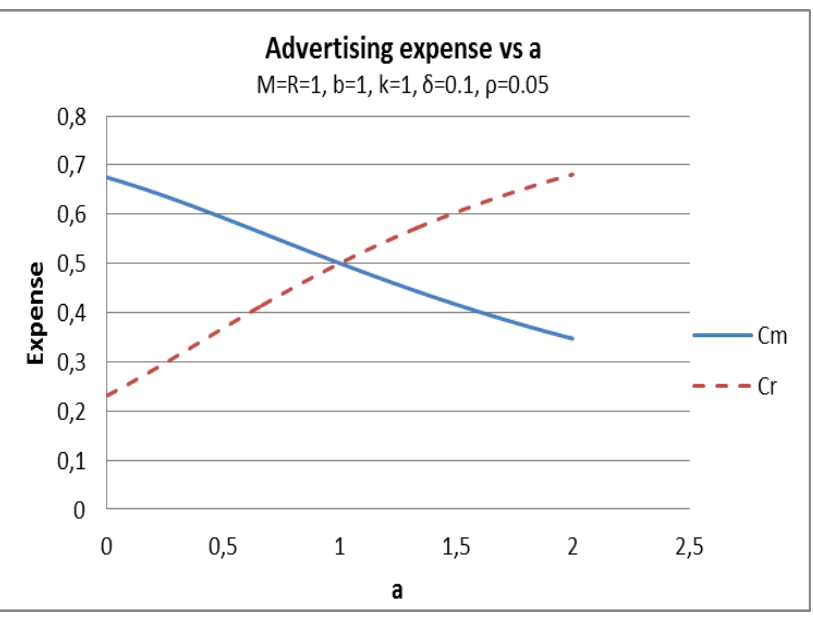

Figure 7: Mfg and ret's total ad. expense vs a

- Impact of advertising effectiveness (Figures 6-17): As the retailer's advertising ef- 


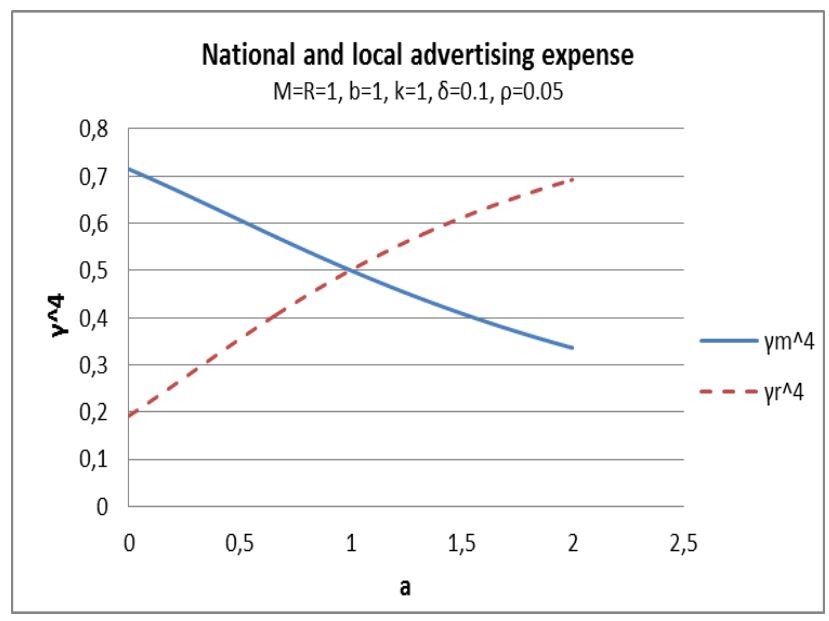

Figure 8: National \& local ad. expense vs a

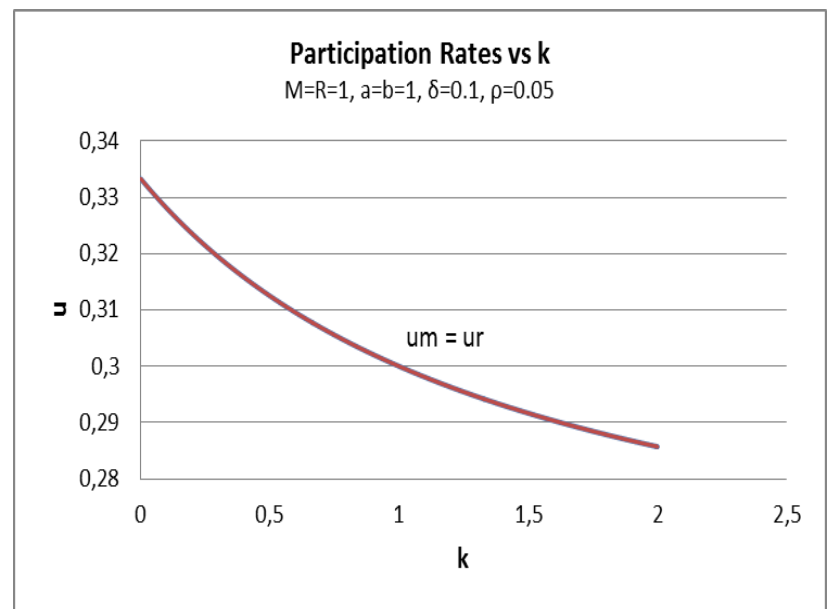

Figure 10: Participation Rates vs k

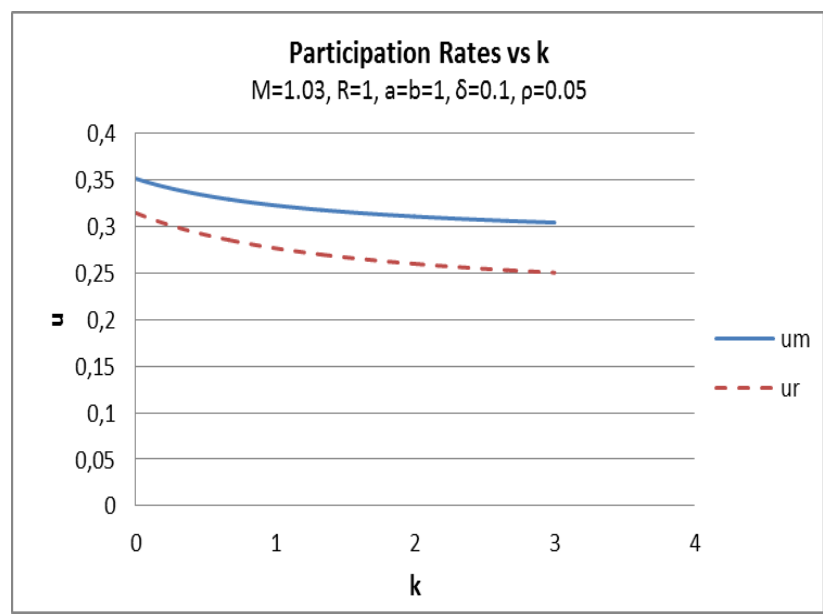

Figure 12: Participation Rates vs k

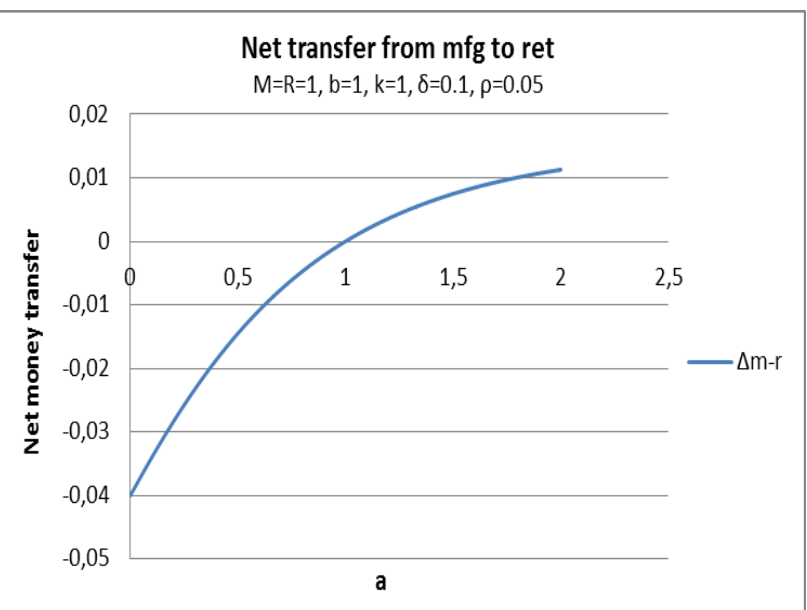

Figure 9: Net transfer from mfg to ret vs a

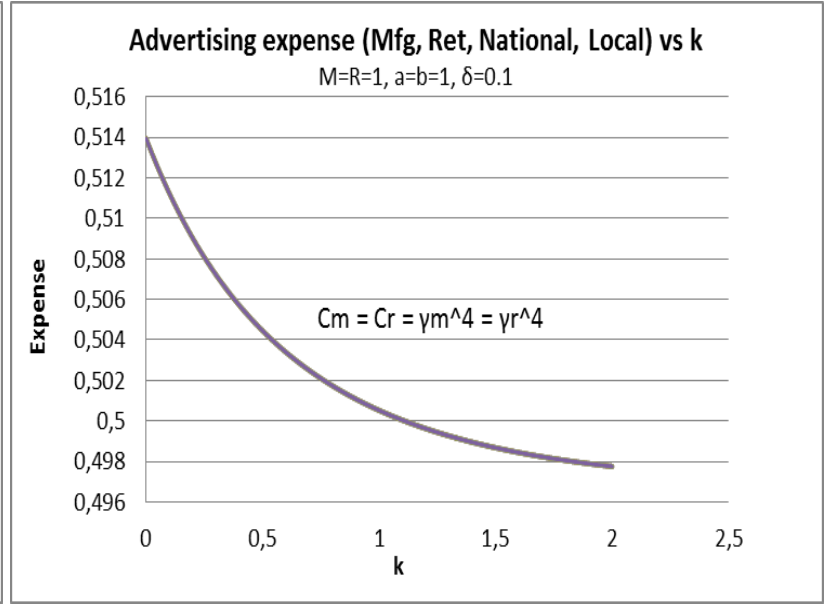

Figure 11: Advertising expenses vs $\mathrm{k}$

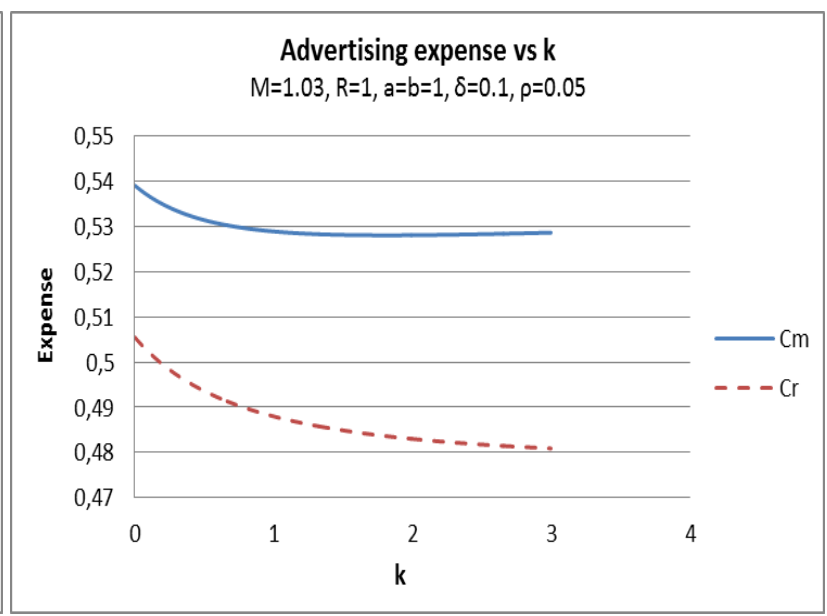

Figure 13: Mfg and ret's total ad. expense vs k 


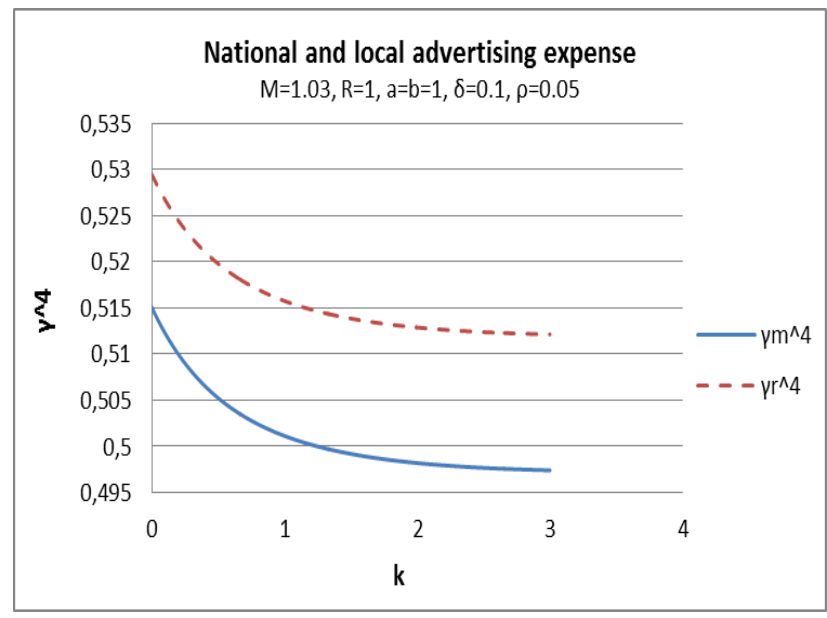

Figure 14: National \& local ad. expense vs $\mathrm{k}$

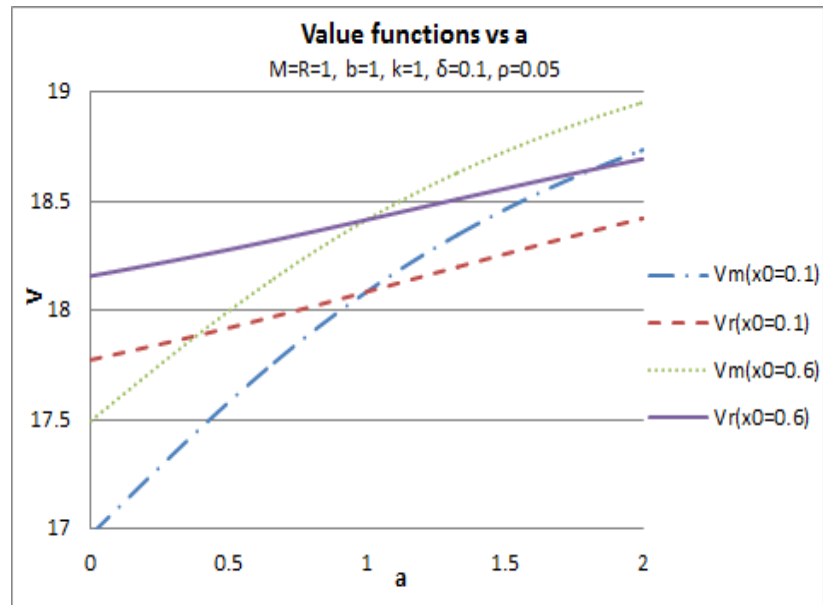

Figure 16: Value function vs a

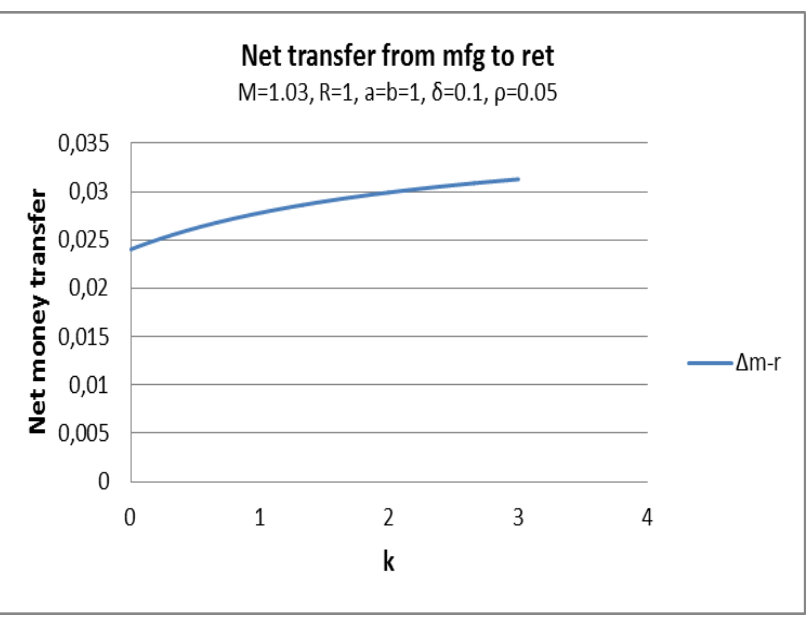

Figure 15: Net transfer from mfg to ret vs $\mathrm{k}$

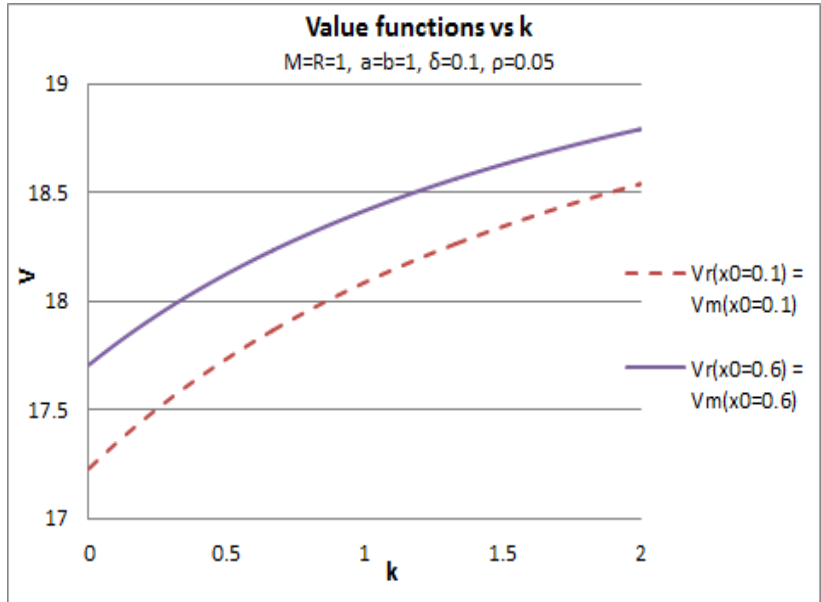

Figure 17: Value function vs $\mathrm{k}$

fectiveness $a$ increases, $u_{r}$, i.e., retailer's advertising support to the manufacturer increases. The retailer's total advertising expenditure and the total local level advertising increase as well. However, the manufacturer's participation rate, his advertising expense, and the total national advertising decrease. Also, the net transfer of money from the manufacturer to the retailer increases as $a$ increases. The net transfer is to the retailer if $a>b$, i.e., when the retailer's advertising effort is more effective than the manufacturer's and to the manufacturer if $a<b$. Symmetric results are obtained when $b$ is changed. On the other hand, as the common advertising effectiveness parameter $k$ increases with $M=R$ and $a=b$, the participation rates of the two channel members are equal and decrease. The national advertising expense of the manufacturer equals the local advertising expense of the retailer and both decrease as the common effectiveness parameter increases. Thus, the net transfer of money between the two 
parties is always zero in this case. However, when $M \neq R$, the participation rates and the advertising expenses, as well as their rates of change are different. For $M>R$, the net transfer of money from manufacturer to retailer is positive and increases as $k$ increases. When $M=R$ and $a=b$ we find that the value functions of both the parties are equal and increase with $k$. Figure 16 shows that the value functions increase as $a$ increases, and are also higher when the initial market share is higher. When $a=b$ and $M=R$, the value functions of both parties are equal and increase as $k$ increases (Figure 17).

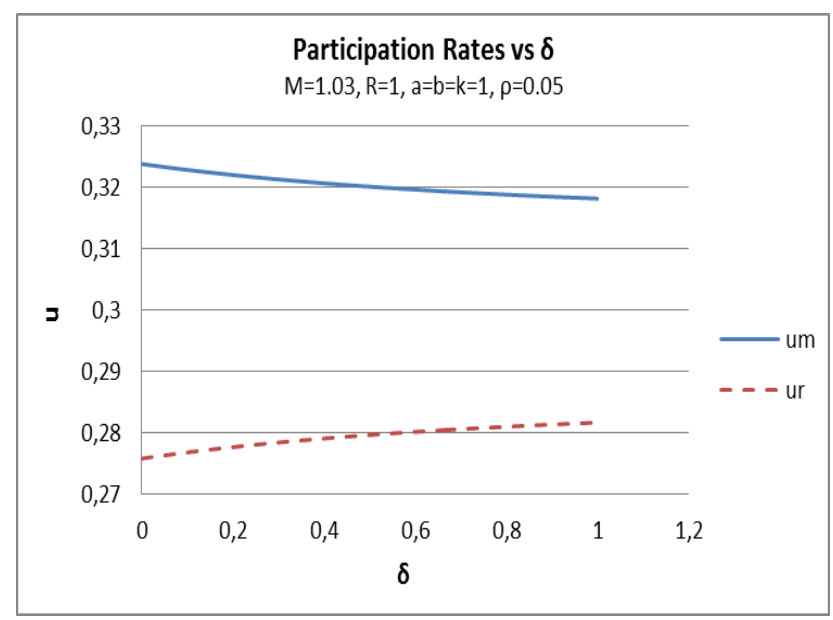

Figure 18: Participation Rates vs $\delta$

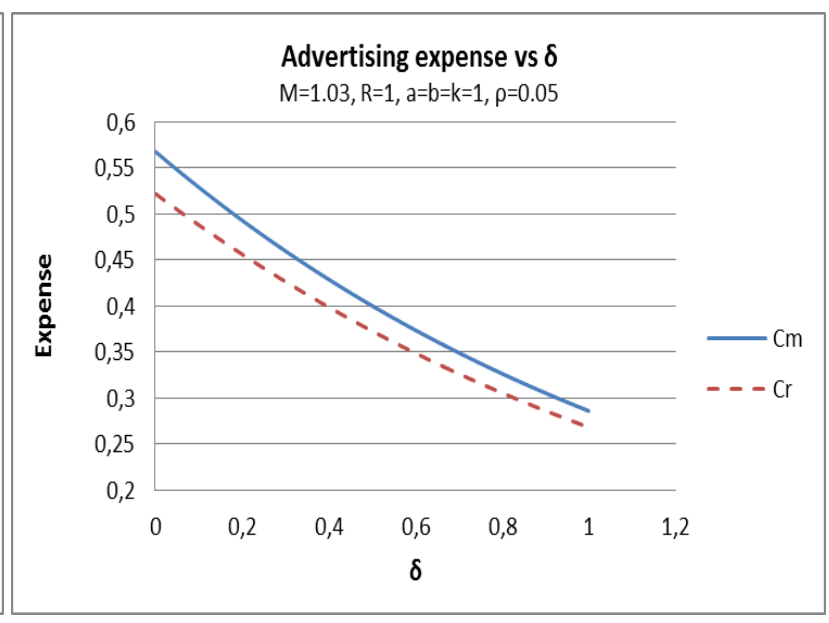

Figure 19: Mfg and ret's total ad. expense vs $\delta$

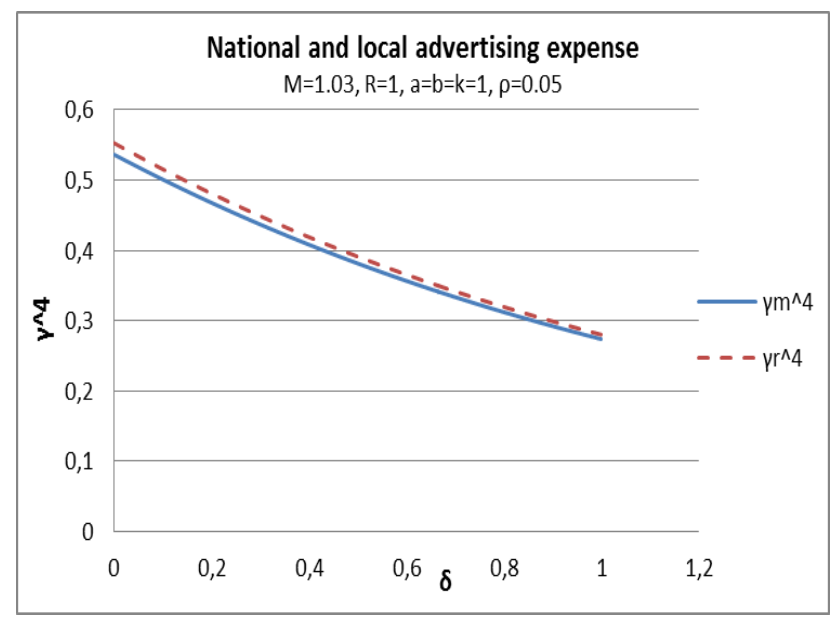

Figure 20: National \& local ad. expense vs $\delta$

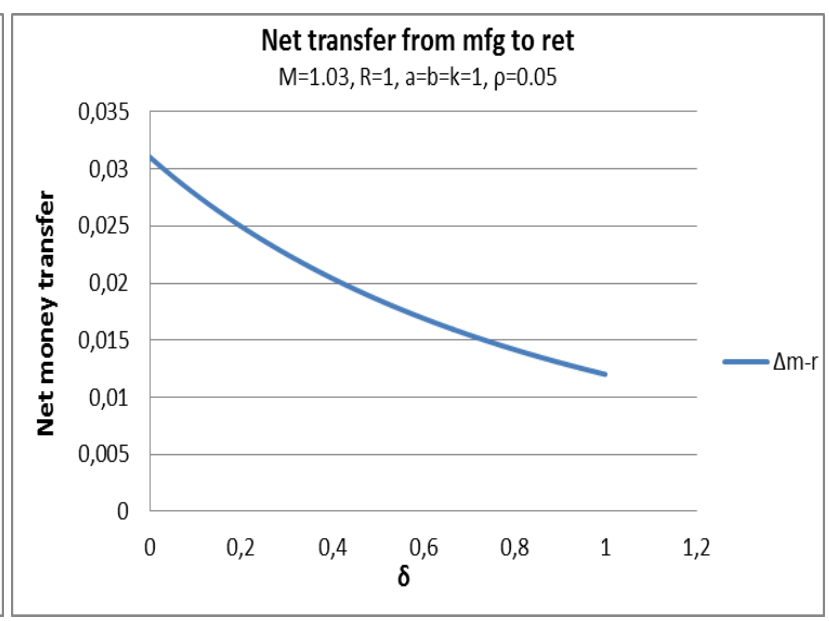

Figure 21: Net transfer from mfg to ret vs $\delta$

- Impact of the decay factor $\delta$ (Figures 18-22) and the discount rate $\rho$ (Figures 24-27): It was observed that when $M=R$ and $a=b$, the participation rate for both members are the same and do not change with $\delta$ or $\rho$. Also, with $M=R$ and $a=b$, we have 


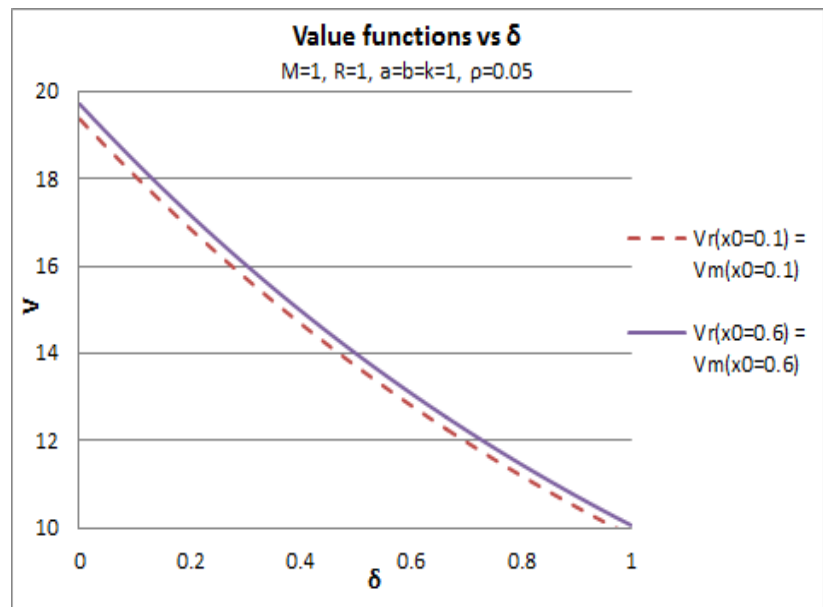

Figure 22: Value function vs $\delta$

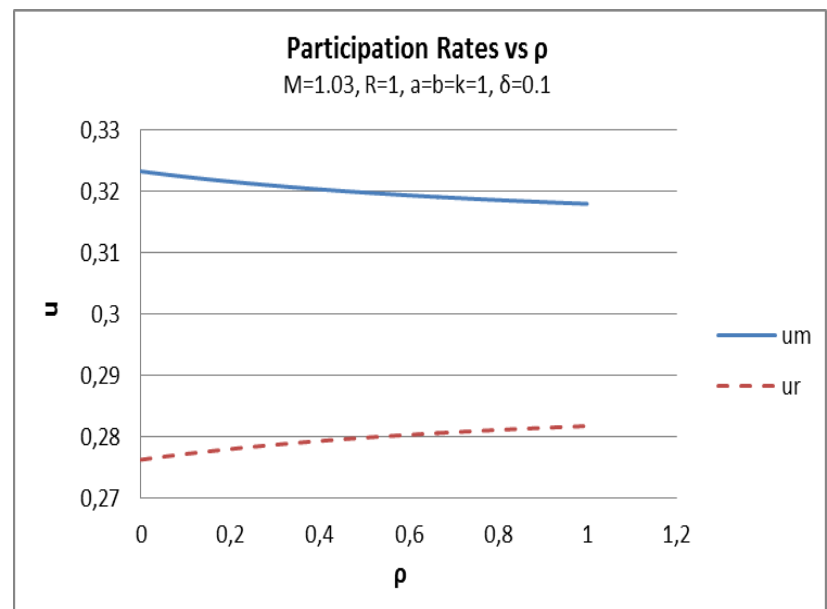

Figure 24: Participation Rates vs $\rho$

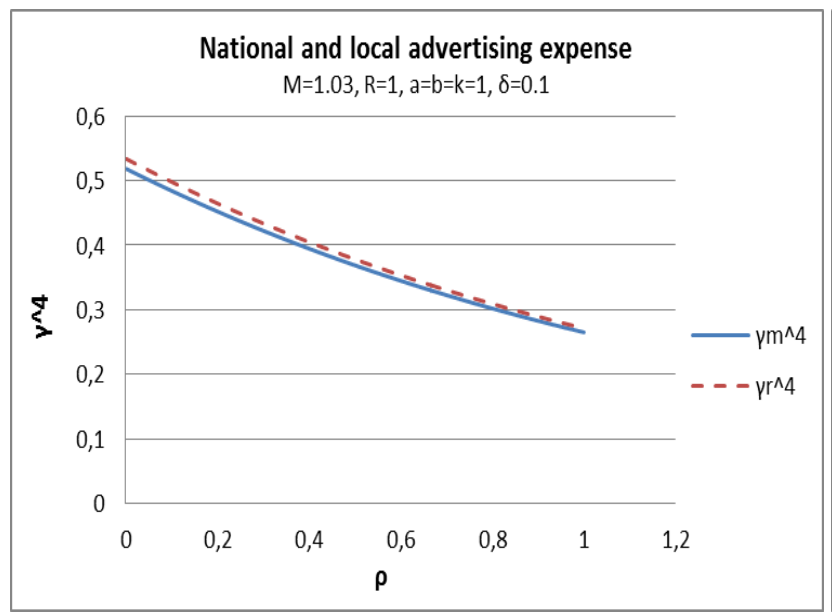

Figure 26: National \& local ad. expense vs $\rho$

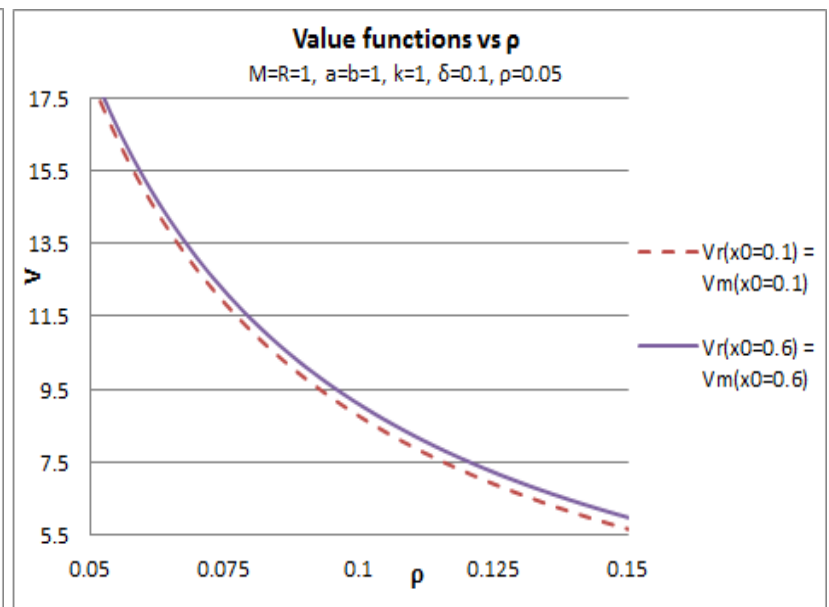

Figure 23: Value function vs $\rho$

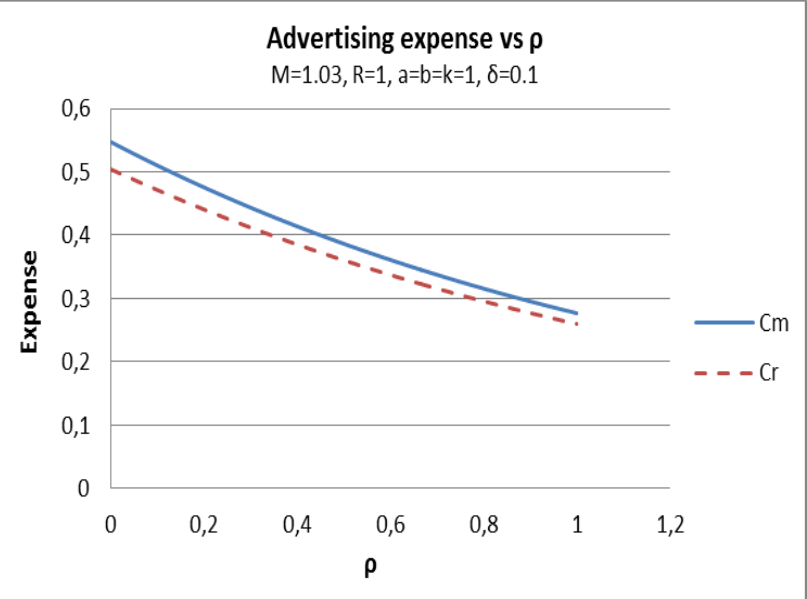

Figure 25: Mfg and ret's total ad. expense vs $\rho$

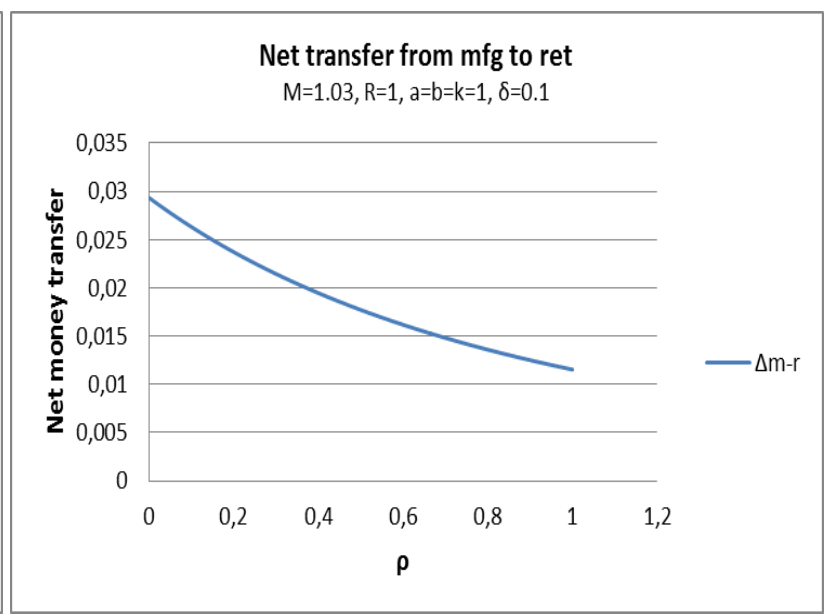

Figure 27: Net transfer from mfg to ret vs $\rho$ 
$C_{m}=C_{r}=\gamma_{m}{ }^{4}=\gamma_{r}{ }^{4}$, and they decrease as $\delta$ or $\rho$ increases. Thus, the advertising expenses are equal and decrease with $\delta$ as well as with $\rho$. The net transfer of money is therefore zero in this case. Figures (18)-(27), on the other hand, show changes in these quantities with $\delta$ and $\rho$, when $M \neq R$. When $M=R$ and $a=b$, the profits of both parties decrease as $\delta$ increases (Figure 22), and also as $\rho$ increases (Figure 23).

\subsection{Comparison with the Traditional Model}

Most game-theoretic models in cooperative advertising consider the scenario in which the mechanism of cooperation is supported by one party to the other, and more specifically, from the manufacturer to the retailer in its promotional efforts. In this paper, using the mixed-leadership framework, we are able to analyze the situation in which each party may offer support to the other in its advertising program. Therefore, it will be interesting to compare the two scenarios, i.e., when there is no clear leader and both may offer support to each other v.s. when one party (manufacturer) is the leader and offers support to the other (retailer). To analyze this, we solved the model by forcing the participation rate $u_{r}(t)$ of the retailer to be zero. Thus, in this case, in the first stage the manufacturer announces his participation rate $\left(u_{m}(t)\right)$ for the retailer, followed by the second stage in which both choose their advertising efforts over time, i.e., $v_{m}(t)$ and $v_{r}(t)$. Figures (28)-(31) present the numerical insights and comparison of the results with the mixed-leadership scenario solved earlier. We find that in the absence of any subsidy offered by the retailer, the manufacturer's participation rate for the retailer is slightly higher as shown in Figure (28), whereas the total advertising expense by both parties are somewhat lower as shown in Figure (29). Figure (30) shows that in the absence of the retailer's support, while the total national advertising expenditure decreases, the total local advertising expenditure increases. Quite obviously, the net transfer from the manufacturer to the retailer is always positive as the retailer does not offer any money to the manufacturer (Figure 31). Figure 32 shows the value functions of the retailer and the manufacturer when $u_{r}=0$, in comparison with the general scenario when both participation rates can be positive. We find that the value functions are slightly lower for both parties when the retailer cannot offer any support to the manufacturer.

\section{Concluding Remarks}

In this paper, we provide a theoretical framework to study a class of stochastic StackelbergNash differential games. We investigate the situation where the roles of two players are mixed: They are leaders on some decisions and followers on the remaining decisions. We establish 


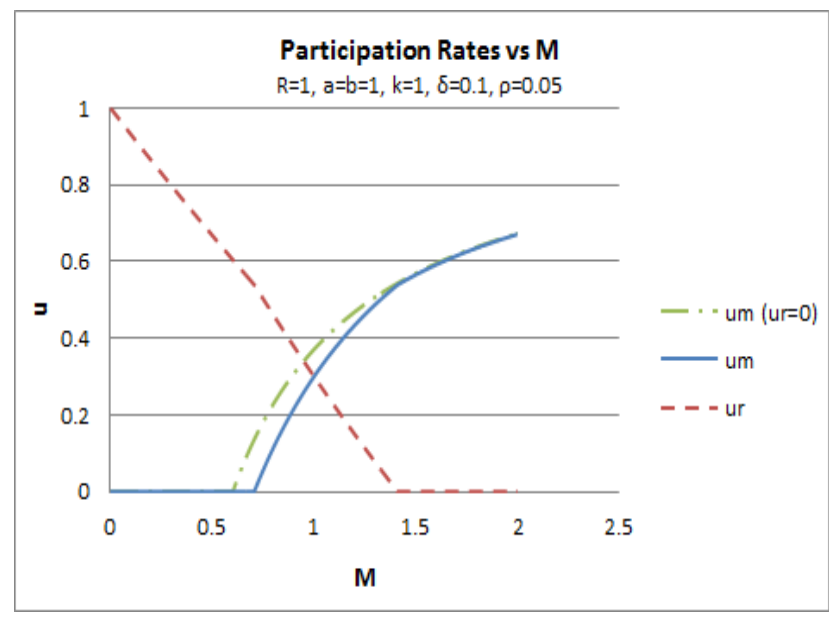

Figure 28: Participation Rates vs $M$ when $u_{r}=0$

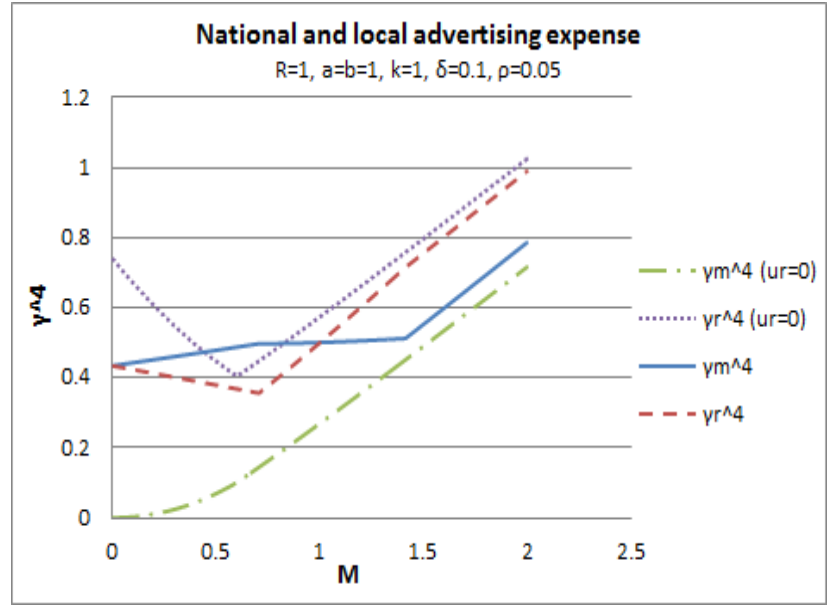

Figure 30: National \& local ad. expense vs $M$ when $u_{r}=0$

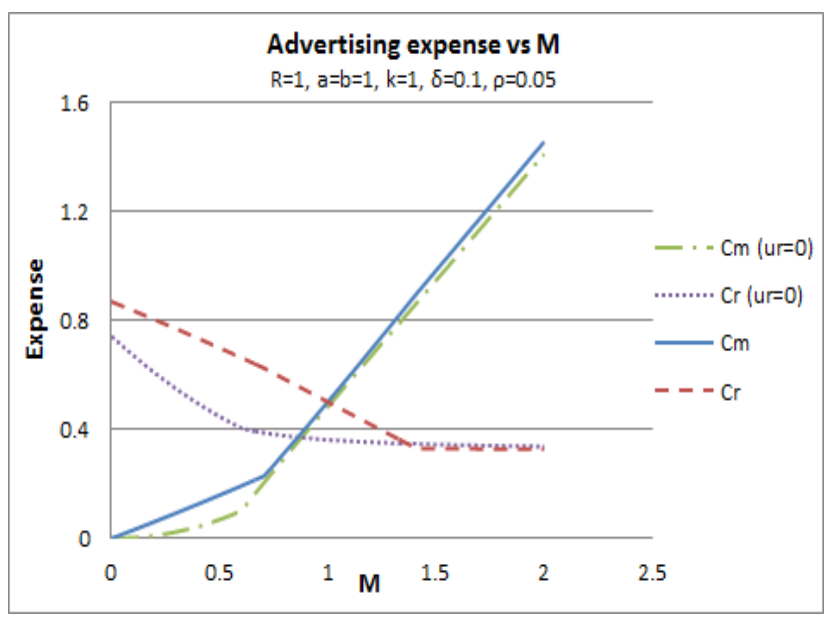

Figure 29: Mfg and ret's total ad. expense vs $M$ when $u_{r}=0$

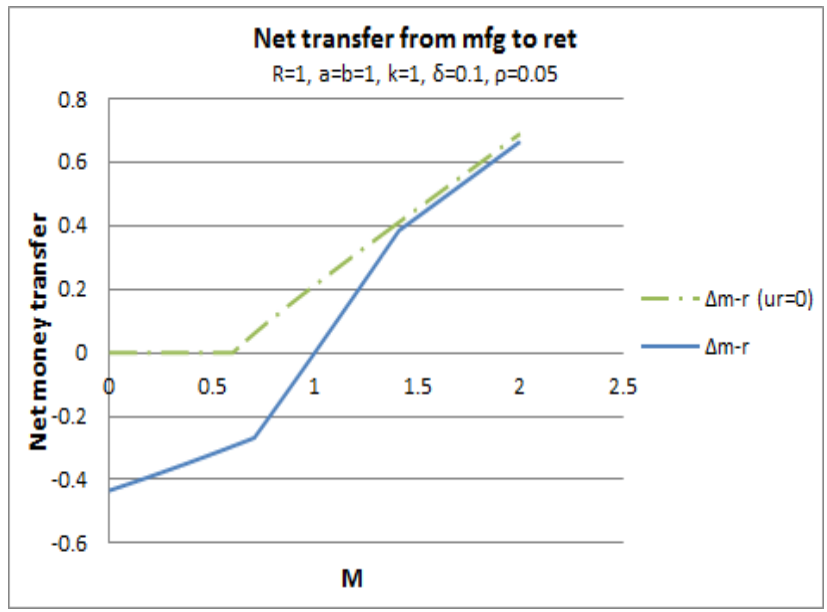

Figure 31: Net transfer from mfg to ret vs $M$ when $u_{r}=0$

a notion of a feedback Stackelberg-Nash equilibrium and prove a verification theorem via dynamic programming, which reduces the task of finding equilibrium strategies in functional spaces to two simple steps. The first steps focuses on solving two static Nash games at the Hamiltonian level in a nested version. The second step solves the corresponding system of HJB equations in terms of the solutions to the two nested Nash games.

While the solutions to the associated HJB equations are difficult to establish in general, we consider a representative example of a mixed leadership game in which solving the associated HJB equations can be reduced to a problem of solving a system of algebraic equations. Specifically, we adopt the mixed leadership game framework to study a cooperative advertising problem in a manufacturer-retailer supply chain which, different from the traditional framework, incorporates the flexibility of the support of the retailer on the manufacturer's 


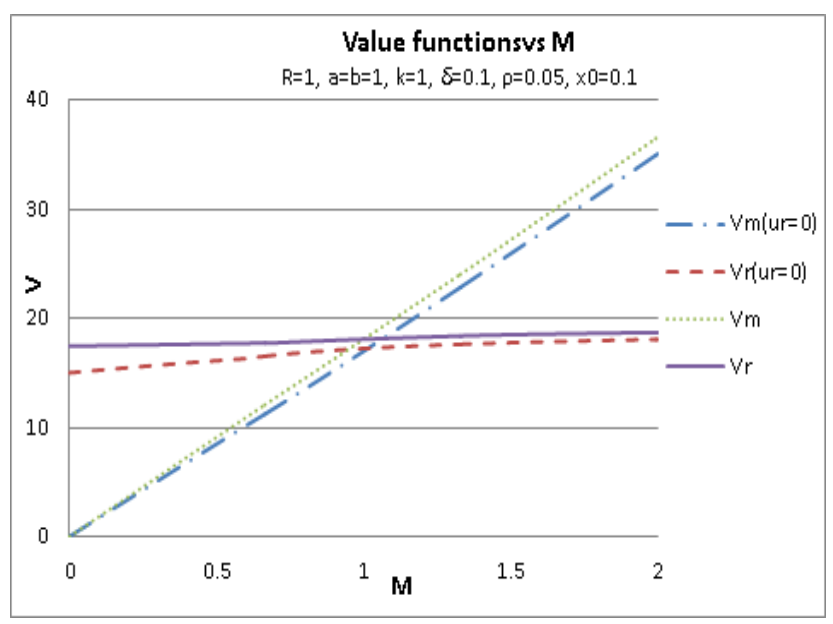

Figure 32: Value functions vs $M$ when $u_{r}=0, x_{0}=0.1$

advertising effort. Based on the verification theorem, we find an equilibrium associated with affine value functions and reduce the problem to a system of quartic algebraic equations. We numerically solve these equations and conduct the sensitivity analysis for the equilibrium behaviors. One interesting thing is that, compared to the traditional cooperative advertising model, mutual supports for the other party's advertising effort benefit both supply chain partners.

The mixed-leadership game framework in this paper suggests several future investigations. Toward the development in cooperative advertising, the mixed leadership game provides the modelling flexibility that allows players to take the mixed leadership roles in different pricing and advertising contexts. Indeed, we have shown that the players in the mixed-leadership games can achieve higher values that those in the traditional cooperative advertising models in this paper. It is therefore imperative to see similar comparisons under different contexts. Secondly, the analysis on the mixed leadership games with controls in the drift and the volatility terms in a high-dimensional setting proves to be another important direction with far-reaching potential applications. Finally, the specific structures in the representative example explored in this paper can also offer new insights into searching for a broader class of mixed game models with tractable, feedback Nash-Stackelberg equilibria. We shall consider these developments in future works.

\section{Acknowledgement}

The authors thank the Editor, Professor G. George Yin, and an anonymous referee for their insightful comments. Special thanks goes to the referee for bringing the interesting works of 
[26], [31], [30], and [32] on the zero-sum stochastic differential games to the authors' attention. Bensoussan acknowledges the financial support from the National Science Foundation under grant DMS-1612880, and the Research Grant Council of Hong Kong Special Administrative Region under grant GRF 11303316. Sethi acknowledges the financial support from the Eugene McDermott Chair Professorship. Siu acknowedges the financial support from the Research Grant Council of Hong Kong Special Administrative Region under grant title: Stochastic Stackelberg Differential Games with Applications in Cooperative Advertising. Yam acknowledges the financial support from HKGRF-14300717 with the project title: New Kinds of Forward-Backward Stochastic Systems with Applications, HKSAR-GRF-14301015 with title: Advance in Mean Field Theory, Direct Grant for Research 2014/15 with project code: 4053141 offered by CUHK. The authors also acknowledge Chi Chung Siu as the corresponding author of this paper.

\section{References}

[1] E. Adida And V. DeMiguel, Supply chain competition with multiple manufacturers and retailers, Oper. Res., 59 (2011), pp. 156-172.

[2] G. Aust And U. Buscher, Cooperative advertising models in supply chain management: A review, European J. Oper. Res., 234 (2014), p. 1-14.

[3] T. BAŞAR AND A. HAURIE, Feedback equilibria in differential games with structural and modal uncertainties, in Advances in Large Scale Systems, J. J. B. Cruz, ed., JAE Press Inc., Connecticut, 1984, pp. 163-201.

[4] T. Başar And G. J. Olsder, Dynamic Noncooperative Game Theory, SIAM Series in Classics in Applied Mathematics, SIAM, Philadelphia PA, 1999.

[5] T. Basar, A. Bensoussan, And S. P. Sethi, Differential games with mixed leadership: The open-loop solution, Appl. Math. Comput., 217 (2010), pp. 972-979.

[6] A. Bensoussan, S. Chen, And S. P. Sethi, Linear quadratic differential games with mixed leadership: The open-loop solution, Numer. Algebra Control Optim., Special Edition to honor Professor George Leitmann, 3 (2013), pp. 95-108.

[7] A. Bensoussan, S. Chen, And S. P. Sethi, The maximum principle for global solutions of stochastic stackelberg differential games, SIAM J. Control Optim., 53 (2015), pp. 1956-1981. 
[8] A. Chutani And S. P. Sethi, Cooperative advertising in a dynamic retail market oligopoly, Dyn. Games Appl., 2 (2012), pp. 347-375.

[9] A. Chutani And S. P. Sethi, Optimal advertising and pricing in a dynamic durable goods supply chain, J. Optim. Theory Appl., 154 (2012), pp. 615-643.

[10] V. DeMiguel And H. Xu, A stochastic multiple-leader stackelberg model: Analysis, computation, and application, Oper. Res., 57 (2009), pp. 1220-1235.

[11] E. J. Dockner, S. Jørgensen, N. Long, And G. Sorger, Differential Games in Economics and Management Science, Cambridge University Press, 2000.

[12] A. Federgruen And M. Hu, Sequential multiproduct price competition in supply chain networks, Oper. Res., 64 (2016), pp. 135-149.

[13] I. I. Gihman And A. V. Skorohod., Stochastic Differential Equations, SpringerVerlag, New York, 1972.

[14] X. He, A. Krishnamoorthy, A. Prasad, and S. P. Sethi, Retail competition and cooperative advertising, Oper. Res. Lett., 39 (2011), pp. 11-16.

[15] X. He, A. Krishnamoorthy, A. Prasad, and S. P. Sethi, Co-op advertising in dynamic retail oligopolies, Decis. Sci., 43 (2012), pp. 73-106.

[16] X. He, A. Prasad, And S. P. Sethi, Cooperative advertising and pricing in a stochastic supply chain: Feedback stackelberg strategies, Prod. and Oper. Manag., 18 (2009), pp. 78-94.

[17] X. He, A. Prasad, S. P. Sethi, and G. J. Gutierrez, A survey of stackelberg differential game models in supply and marketing channels, J. Syst. Sci. Syst. Eng., 16 (2007), pp. 385-413.

[18] R. IsAacs, Differential games: Their scope, nature, and future, J. Optim. Theory Appl., 3 (1969), pp. 283-295.

[19] S. Jørgensen, S. P. Sigué, And G. Zaccour, Dynamic cooperative advertising in a channel, J. Retail., 76 (2000), pp. 71-92.

[20] S. Jørgensen, S. TABoubi, And G. ZacCour, Cooperative advertising in a marketing channel, J. Optim. Theory Appl., 110 (2001), pp. 145-158.

[21] S. Jørgensen, S. TABoubi, And G. Zaccour, Retail promotions with negative brand image effects: Is cooperation possible?, European J. Oper. Res., 150 (2003), pp. 395-405. 
[22] S. Jørgensen And G. Zaccour, A survey of game-theoretic models of cooperative advertising, European J. Oper. Res., 237 (2014), p. 1-14.

[23] S. Karray and G. ZACCOUR, A differential game of advertising for national brand and store brands, in Dynamic Games: Theory and Applications, A. Haurie and G. Zaccour, eds., Springer, Berlin, 2005, pp. 213-229.

[24] T. Li And S. P. Sethi, A review of dynamic stackelberg game models, Discrete Event Dyn. Syst.-B, (2017).

[25] B. Øksendal, L. Sandal, And J. Uboe, Stochastic stackelberg equilibria with applications to time dependent newsvendor models, J. Econom. Dynam. Control, 37 (2013), pp. 1284-1299.

[26] H. PhAm AND J. Zhang, Two person zero-sum game in weak formulation and path dependent bellman-isaacs equation, SIAM J. Control Optim., 52 (2014), pp. 2090-2121.

[27] S. P. SETHI, Deterministic and stochastic optimization of a dynamic advertising model, Optimal Control Appl. Methods, 4 (1983), pp. 179-184.

[28] H. Sherali, A. Soyster, And F. MurPhy, Stackelberg-nash-cournot equilibria: Characterizations and computations, Oper. Res., 31 (1983), pp. 253-267.

[29] S. Sigué and P. Chintagunta, Advertising strategies in a franchise system, European J. Oper. Res., 198 (2009), pp. 655-665.

[30] M. SîRBU, On martingale problems with continuous-time mixing and values of zero-sum games without the isaacs condition, SIAM J. Control Optim., 52 (2014), pp. 2877-2890.

[31] M. SîRBU, Stochastic perron's method and elementary strategies for zero-sum differential games, SIAM J. Control Optim., 52 (2014), pp. 1693-1711.

[32] M. SîrBu, Asymptotic perron's method and simple markov strategies in stochastic games and control, SIAM J. Control Optim., 53 (2015), pp. 1713-1733.

[33] H. von StackelBerg, Marktform und Gleichgewicht, Springer, Vienna, 1934.

[34] D. Wolf And Y. SmeERs, A stochastic version of a stackelberg-nash-cournot equilibrium model, Manag. Sci., 43 (1997), pp. 190-197. 